\title{
Simulation of Rough Surfaces and Analysis of Roughness by MATLAB
}

\author{
F. Gascón ${ }^{1}$ and F. Salazar ${ }^{2}$ \\ ${ }^{1}$ Departamento de Física Aplicada II. ETS Arquitectura (US). \\ Avda. Reina Mercedes 2, 41012 Sevilla \\ ${ }^{2}$ Departamento de Física Aplicada. ETSI Minas (UPM). \\ C/Ríos Rosas 21, 28003 Madrid \\ Spain
}

\section{Introduction}

The simulation of physical phenomena in science and engineering has become an important tool because it allows studying a wide range of real problems. On the other hand, it allows resolving problems that, because of its difficulty, it would be not possible to solve by analytical methods. Moreover, simulation is fast and versatile since it permits to vary parameters of the problem easily, allowing analyzing the effect of the modification of them in the response of the system examined.

Simulation requires programming, for which there are many different languages. Each of them has a particular internal structure that distinguishes it from others. Therefore, depending on the problem to be study, it may be advisable to use a specific programming language.

In the scientific-technical context MATLAB has been increasingly used by the great advantages that it offers. For example, the instructions are interpreted and not compiled, the user to enter commands interactively. The data processing is flexible. They can be read and stored in two different formats, ASCII and MATLAB format. ASCII has the advantage that the data and results may be used for other programs. However, MATLAB format may be faster. On the other hand, many functions and libraries of MATLAB are written MATLAB language, enabling the user access to the source files. It is possible to execute instructions of the operating system without exiting the program. Moreover, this language is portable in platforms as Windows or Apple, commonly employed by the researcher. From the point of view of numerical calculation, the use of matrices as basic elements makes it efficient and easy to employ, being also possible to perform graphics of curves and surfaces. Finally, the operations can be performed with simple and intuitive expressions similar to those used in science and engineering.

MATLAB has been used for many applications in general physics, mathematics, optics, electronics, chemistry, biology, medicine, and artificial intelligence, among others. Now we want to employ MATLAB to simulate an optical procedure to measure surface roughness. Thus, the aim of this paper is the determination of the roughness of a surface from the analysis of the speckle pattern obtained in the far field, when the object is illuminated with a monochromatic beam perpendicularly to its surface. 
The surface analysis of materials is of great importance, since many technological problems require, previously, the study of the surface state. One of the parameters of any material that changes easily with time is the roughness. Indeed, in many sectors, as civil engineering, architecture, mechanical engineering, etc. materials of different forms and properties are commonly employed, which must meet certain requirements to ensure their use. For this reason, the measurement of some surface parameters, as roughness, must be taking into consideration.

There are different methods for determining roughness. One of the most employed is the profilometer (see next section). However, this paper deals with an optical method based on the speckle interferometry which has some advantages. The methodology is fast, accurate, and does not contact the specimen. Above all this we will talk in the next sections

\section{Discretization of the problem. Roughness}

A classic device for measuring surface roughness is the mechanical profilometer which is formed by a tiny stylus (with a small ball), and a displacement sensor. The typ moves along a straight line parallel to the surface plane and records the displacements in the perpendicular direction, tracing out the outline of the surface. If the ball has a diameter $b d$ it can not be inserted between two grooves whose distance is less than $b d$, being only possible to detect the topographic level with a distance between grooves greater than $b d$.

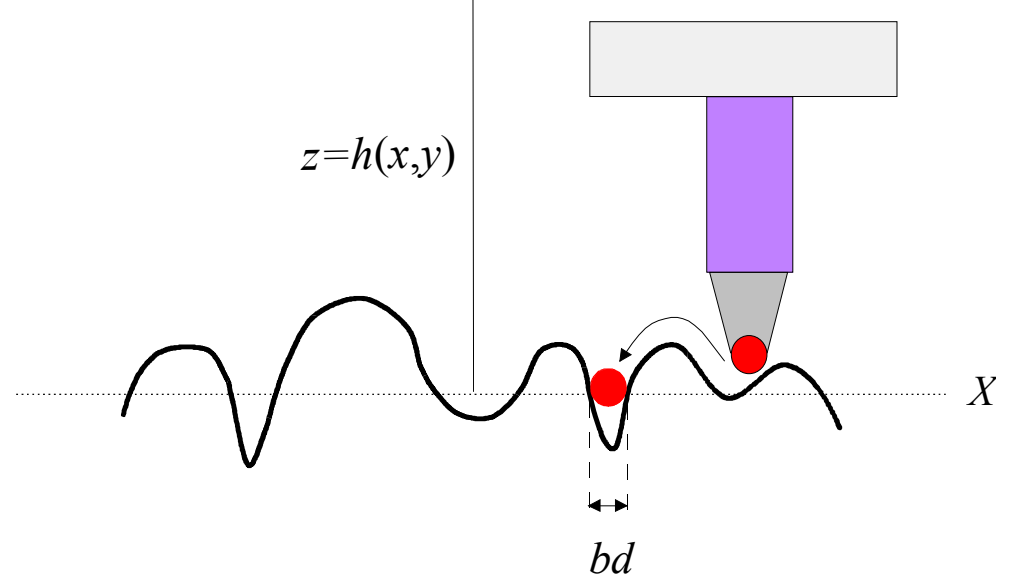

Fig. 1. Classical device for measuring roughness. Observe that when the diameter of the end needle $b d$ is greater than the groove, the transducer can not reproduce the high frequencies of the surface outline.

In this article we are interested in using a speckle technique to measure the roughness of a surface. From a didactic viewpoint, the explained idea of the profilometer may be employed to understand the sampling, when a rough surface is simulated by MATLAB.

To start let us suppose a one-dimensional rough surface, and then we extend the results to the case of two variables.

The height of the rough surface can also be measured by sampling. With this aim let us consider a curve $z=h(x)$ as shown in Fig. (2), aligned on the $O X$ axis. For sampling the 
function $h(x)$ the $X$ co-ordinate axis is divided into intervals of length $u$ measured with respect the origin $O$ resulting in a system of aligned points of co-ordinates $0, u, 2 u, 3 u, \ldots$ The distance $u$ between two neighbor points, i.e. the sample interval, is called the sampling period and its value is chosen depending of the function to be investigated (in our case the form of the surface). The distance $u$ between two points may be likened to the ball diameter $b d$ of the profilometer.

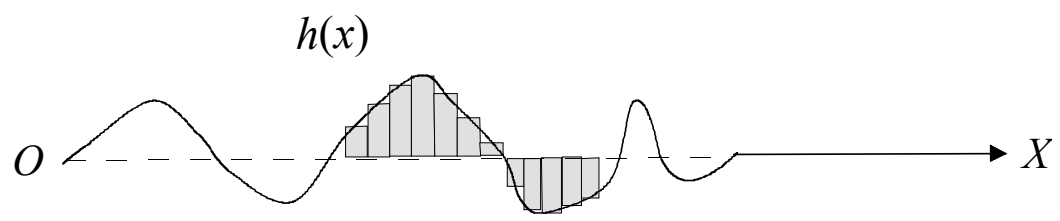

Fig. 2. Rough surface represented by $z=h(x)$. If the sampling period is chosen small, the discrete function $h(x p)$ is very similar to the actual surface.

If $N$ samples are taken, they form a string of $N$ integers for which a value $z_{p}=h(x p)$ is given. This set of numbers is collecting in a matrix IF of dimension $N \times 1$. The range of variation of the index $p$ is $1,2,3, \ldots, N$, and it represents the element $p$ of the string. Therefore this index $p$ is related with the sampling period as follows: $x=0, u, 2 u, 3 u, \ldots,(p-1) u$. Two neighboring elements of the IF matrix contain the values of the surface heights of the grooves of two points on the reference plane separated $u$ meters.

As it will see, when studying the phenomenon of diffraction in the far field, the Fourier transformation must be applied. Therefore, we need to study also the sampling in the frequency domain.

When calculating the finite discrete Fourier transform (DFT) of the IF matrix of $N$ elements, a new set of $N$ numbers is obtained which is grouped in another matrix $F O$ of dimension $N \times 1$. Due to the Fourier transform is performed from the discrete values of $I F$, the result is also discrete. As a result the distance between two points of the transformed numbers in frequency domain is also quantified. Denoting by $\mathrm{v}(1,2, \ldots N)$ the index for the matrix $F O$, the row index represents the harmonic components whose frequencies are $\alpha=0,1 /(N u), 2$ $/(N u), \ldots,(\mathrm{v}-1) /(N u)$. The sampling frequency is defined as $f_{\mathrm{s}} \equiv 1 / u$, measured in $\mathrm{m}^{-1}$, and represents the number of measures per unit length. By using this expression, spatial frequency components may also be written in the form $\alpha=0, f_{\mathrm{s}} / N, 2 f_{\mathrm{s}} / N, \ldots,(v-1) f_{\mathrm{s}} / N$. In general, the matrix element $\mathrm{v}$ of $F O$ represents the harmonic $\alpha_{v}$ in the space of frequencies

$$
\alpha_{v}=\frac{(v-1)}{N u}
$$

Thus there is a correspondence between the index $v=1,2,3, \ldots, N$, and the spatial frequency $\alpha$ by means of the factor $\left(f_{\mathrm{s}} / N\right)$. Obviously, the sampling process implies that some information about the sampled function is loosed, because no value between two neighboring points is known. However, depending on the physical problem studied, using the Shannon theorem, the sample interval can be modified so that the data be enough for numerical calculus.

Taking into account that the expression obtained for representing a point on the $O X$-axis has the form $(p-1) u$, it seems to be appropriate to change to non-dimensional variables. To do so 
we divide $x$ for $u$ resulting $(x / u)=(p-1), p$ being $1,2, \ldots, N$. This new variable represents the distance from the origin $O$ to an arbitrary point on $O X$ (Fig.3), measured in units of the sampling period $u$, and the elements of the matrix IF the surface heights at each point. The same idea applies to frequency domain.

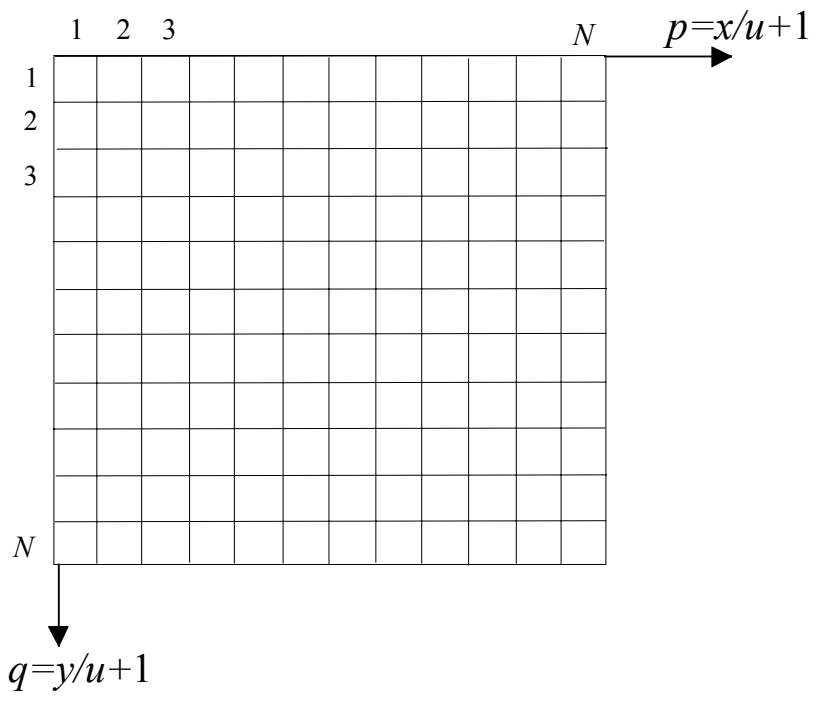

Fig. 3. Reference system without dimensions. The numbers represent the co-ordinates of the matrix elements. At each point $(p, q)$ we assign the corresponding value of the surface height.

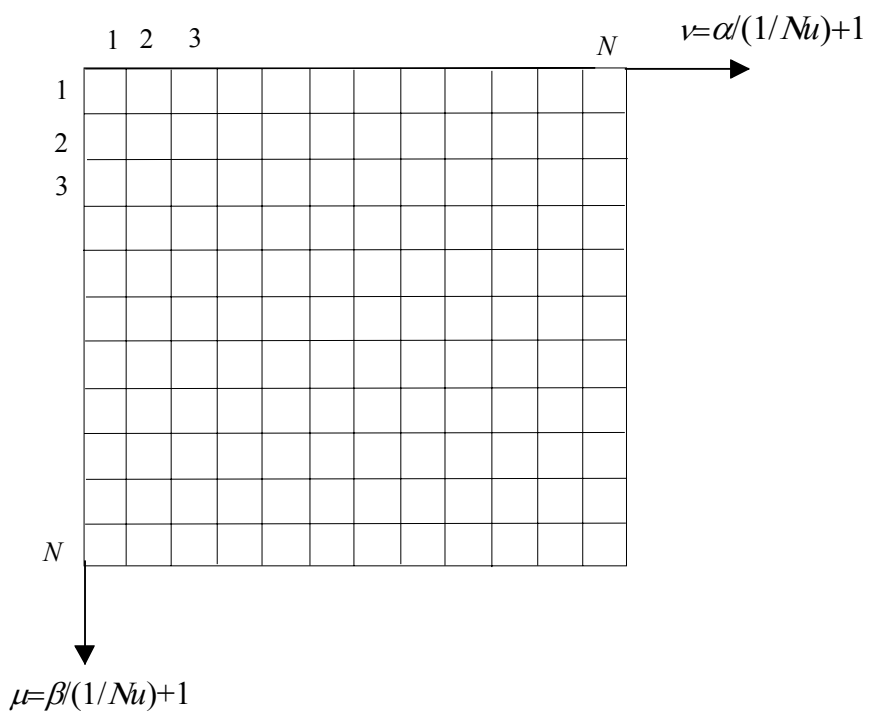

Fig. 4. Space of non-dimensional frequencies. Observe that this grid is initially determined by the matrix $I F$, then it also has $N x N$ elements 
By setting $\alpha(N u)=\alpha /(1 / N u)=(v-1)$ the spatial frequency $\alpha$ is converted in a dimensionless number representing the basic unities of measurement in this space. Furthermore, $\alpha /(1 / N u)$ is directly the frequency measured in unities of $(1 / N u)$ corresponding to the element $v$ of the matrix FO (Fig.4).

When generating a rough surface the components of IF are real numbers (Fig.3). However, as we will see, the diffraction of a light beam by a surface can introduce phase factors resulting in complex numbers in the elements of matrix IF. In any case the resulting $N^{\times} 1$ string of IF and its fast Fourier transform (FFT), are calculated without difficulty with MATLAB. One advantage of the aforementioned procedure is that the sampling distance $u$ between two points of IF is not directly involved in the numerical calculation, and then it may be considered as a parameter. For this reason the DFT and the FFT of $I F$, i.e. FO, is universal respect to the parameter $u$, because the components of $F O$ depend only of the nondimensional elements of the matrix IF.

Although with the change of variables introduced the components of IF and FO are dimensionless, they have physical meaning. In the present study the $p$ element of $I F$ is a measure of the height of the point at position $x=(p-1) u$. FO may be interpreted in the same way. So setting any number to the sampling period $u$ the values obtained for $F O$ show the harmonic amplitudes. For example, giving $u$ the arbitrary value $10^{-4} \mathrm{~m}$, and choosing $N=64$, we have for the first non-zero frequency components, $\alpha=156.2,312.5,468.8, \ldots \mathrm{m}^{-1}$.

The above is easily applied to a two-dimensional simulation. For this let us consider two coordinates $(x, y)$ of the system OXY (Figs.3,4). For each point of this reference plane is assigned a value which corresponds to the surface height at this point. The data are placed in a two-dimensional array IF. If the sampling is done with the same number of samples, say $N$, the dimension of the matrix IF is $N^{\times} N$ (Fig. 3). Two points of coordinates $\left(x_{\mathrm{i}}, y_{\mathrm{j}}\right)$ and $\left(x_{\mathrm{k}}\right.$, $\left.y_{1}\right)$ respectively are separated in the matrix IF a distance $\left((i-j)^{2+}(k-l)^{2}\right)^{1 / 2} u$, and in dimensionless co-ordinates $\left((i-j)^{2}+(k-l)^{2}\right)^{1 / 2}$. In relation to $F O$ similar expressions may be obtained, but in frequency space. So the spatial frequencies between two points whose coordinates are $\left(\alpha_{h}, \beta_{k}\right)$ and $\left(\alpha_{l}, \beta_{m}\right)$ is $\left((h-k)^{2+}(l-m)^{2}\right)^{1 / 2}(1 / N u)$ in $\mathrm{m}^{-1}$, and without dimensions $\left((h-k)^{2+}(l-m)^{2}\right)^{1 / 2}$.

\section{Fraunhofer diffraction with MATLAB}

In this section we are interested in the phenomenon of diffraction of light, given the importance to understand the speckle patterns. With this objective let us use the experimental lay-out depicted in Fig.(5). A collimated monochromatic laser beam LB of wavelength $\lambda$ is directed to a beam splitter BS, which projects the light perpendicularly on a diffracting rough sample $S$ located on the $O X Y$ plane. The surface has, in principle, a variable reflectance $R(x, y)$. This means the different scatters that form the surface may have distinct reflection properties. The shape of the radiation beam used determines the geometry and the intensity inside of the illuminated area. If we suppose a beam of homogeneous intensity, its geometry can be expressed easily (in view of the simulation) as an opaque mask $M$ placed on the surface, which has the function to define the illuminated area (Fig. 6). Taking into consideration the most cases studied in optics, we will choose a circular mask of diameter $D$. An observation screen is placed parallel to the diffracting surface at a distance of $z$ from its plane. The points on the observation plane are specified by means of its $x^{\prime}, y^{\prime}$ coordinates, with respect to an $O^{\prime} X^{\prime} Y^{\prime}$ coordinate system (on the CCD camera). 


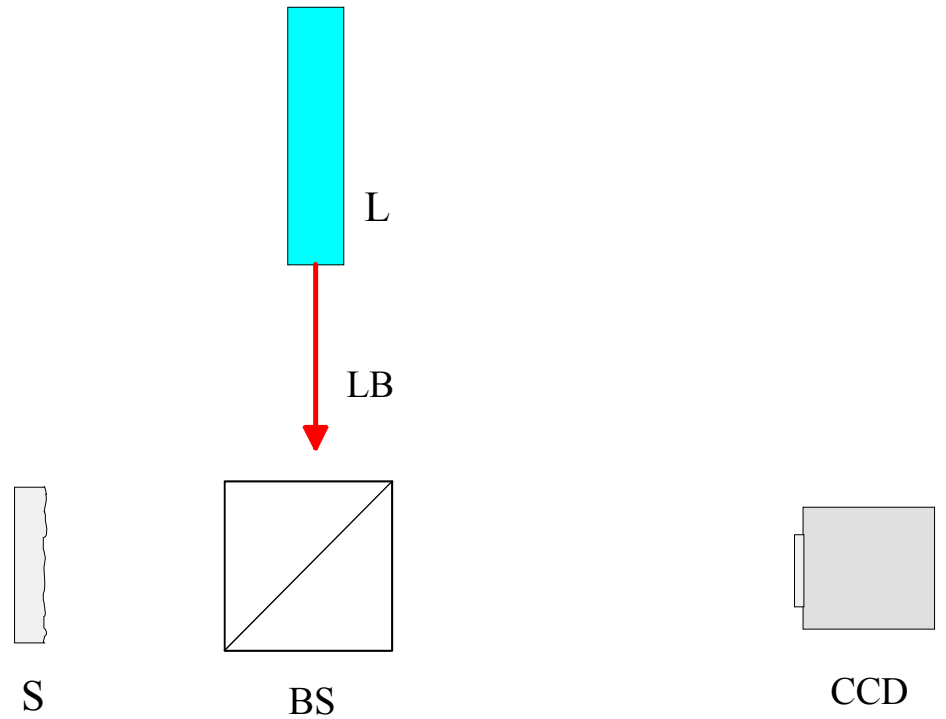

Fig. 5. Experimental set-up. L, laser; LB, laser beam; S, rough sample; BS, beam splitter; CCD camera.
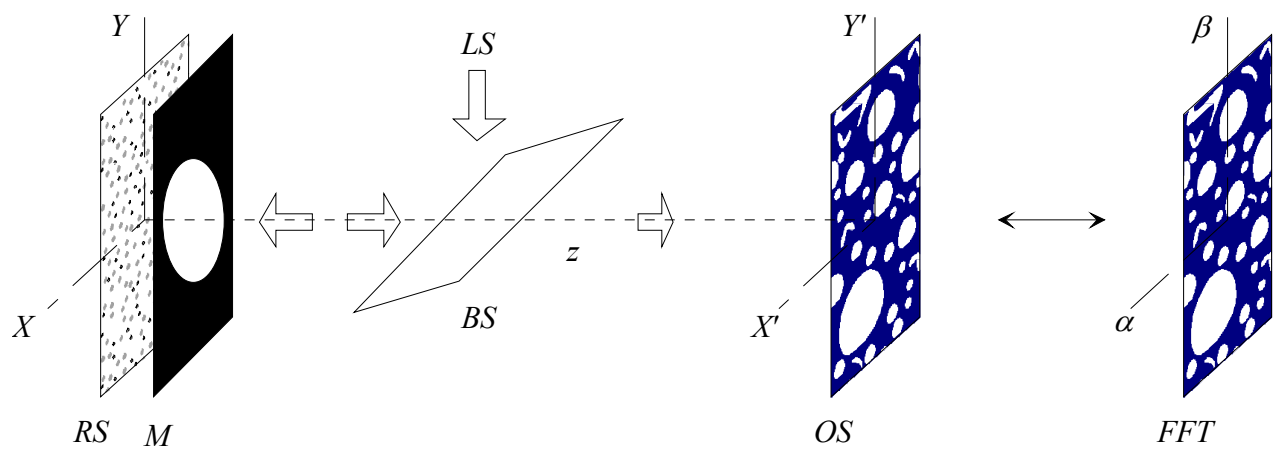

Fig. 6. Steps employed for simulating the rough surface, the aperture, and the speckle pattern. $R S$, rough surface; $M$, mask; $B S$, beam splitter; $F O$, matrix which elements represent the Fourier transform of the reflectance $R(\mathrm{x}, \mathrm{y})$ on the $O X^{\prime} Y^{\prime}$ reference system. The intensity $O S=|F O|^{2}$ can be interpreted also as an angular spectrum $(\alpha, \beta)$.

Supposing that the scalar diffraction theory applies, the Fresnel-Kirchhoff integral and the theories of Rayleigh-Sommerfeld can be used. However, the calculation of the diffraction pattern through these theories is not always easy to carry out. Sometimes the procedure may be simplified under certain conditions of the problem. So if the linear dimensions of the aperture (mask in our case) is much greater than the wavelength, i.e. $\mathrm{D}>>\lambda$, and the distance $z$ between the surface and the observation plane is great enough, the paraxial theories apply. In this case, the mathematical expression for the diffracted field depends on the specific 
dependence between $D$ and $z$. When expanding the phase term in the Fresnell-Kirchhoff integral is not possible to neglect the quadratic terms that appear we speaks of Fresnel diffraction. On the contrary, if these terms can not be tacked into consideration we have Fraunhofer diffraction. These approximations are the most important cases in the field of the classical optics.

A possible quantitative criterion to be employed in order to use the Fraunhofer approximation, or that of Fresnel, is based on taking a circle of diameter $D$, which only includes the regions of interest (in the present case the hole of the mask). Let $r$ be the distance from a point on the diffracting surface to the observation point. Let $\rho$ be the distance from the centre of the circle to a point inside its circle. If $2 \pi r / \lambda$ varies linearly with $\rho$, the diffraction is called Fraunhofer diffraction; if the variation has non-linear terms of magnitude comparable with $\pi / 2$, the diffraction is said Fresnel diffraction. Therefore, for Fraunhofer diffraction we obtain $z>>D^{2} /(4 \lambda)$. In short, the diffracting area must be greater than $\lambda$ and the observation of the intensity pattern must be carried out from a large distance with respect to the scatter surface. In other circumstances, i.e. if the distance $z$ does not fulfil the conditions needed, non-paraxial terms of the phase must be included in the integrand of the Fresnell-Kirchhoff integral (higher expansion coefficients).

Fraunhofer diffraction is related with the Fourier transform which takes an angular spectrum of the reflectance (or transmittance) to be considered. From a physical point of view it is equivalent to observe the phenomenon in the far field (another possibility is to employ a lens and locate the observation plane on its back focal plane). This angular spectrum means that the Fraunhofer diffraction gives the behaviour of the field amplitude for the directions in space. If we use two variables, the amplitude of the diffracted field done through the Fourier transform depends on $\alpha$ and $\beta$, which are related with the directions $\left(\theta_{x}, \theta_{y}\right)$ through the following expressions

$$
\alpha=\frac{\cos \theta_{x}}{\lambda}, \beta=\frac{\cos \theta_{y}}{\lambda} .
$$

As we will see in the following section, the proposed method for measuring roughness is developed under the supposition that the conditions of the Fraunhofer diffraction apply. Therefore, this case must be translated to the context of MATLAB.

With this aim, the basic results of the preceding section should used. The elements $f_{o_{\mathrm{ij}}}$ of the matrix $F O$ belonging to a row or column represent the complex amplitude of two harmonics separated $1 /(N u)$. Therefore the first angular direction is $\theta_{x}=\cos ^{-1}\left((v-1)\left(\lambda f_{s} / N\right)\right)_{v=1}=\frac{\pi}{2}$, which corresponds to the frequency $\alpha=0$ and the direction for the least coefficient of $F O$ is $\theta_{\mathrm{x}}=\cos ^{-1}\left[(N-1) \lambda f_{s} / N\right]$ corresponding to the higher frequency $\alpha=(N-1) f_{s} / N$. In the case of nondimensional variables we can use for the two axes $N u \cos \theta_{x} / \lambda$, and $N u$ $\cos \theta_{y} / \lambda$, respectively. If the diffraction pattern is observed on a plane screen a distance $z$ from the diffusing surface, the spatial frequencies may be related with points on that plane. For small angles $\theta$ it can be written:

$$
\alpha=\frac{\cos \theta_{x}}{\lambda} \approx \frac{x^{\prime}}{\lambda z}
$$


and

$$
\beta=\frac{\cos \theta_{y}}{\lambda} \approx \frac{y^{\prime}}{\lambda z} .
$$

Due to the properties of the Fourier transform, the FFT of the reflectance will contain N/2 of positive frequencies, and N/2 negative, whose zero spatial frequency occurs at $v=1$. In the FFT, the independent variable is the frequency, and in the representation with positive and negative frequencies its maximum value will be $f_{s} / 2$. Based on a reflectance matrix of $N x N$ elements located at the XOY axes associated, practically centred in the middle, we calculate FO by means of the FFT, obtaining another matrix from the centre of which the amplitude of the null frequency harmonic component is indicated.

The intensity registered over a direction $(\alpha, \beta)$ is found by computing the square modulus of the Fourier transform (FT) of the scattering surface delimited by the mask $M$, after centring the FFT by means of the command $C(i, j)=(-1)^{(i+j)}$.

\section{Speckle pattern generation}

When a laser beam illuminates a rough surface at scale of the wavelength, the diffraction pattern consists of a random distribution of intensity called speckle. The apparition of speckle may be understood by the fact that the coherent waves falling on the rough surface travel a different optical path from the diffusing surface to the observation point. When the object is rough, the reflectance is a random function on the aperture, and then the corresponding optical paths for the different scatters vary rapidly. As a result, the intensity on the observation screen (or space) also varies very quickly from one point to another of its surroundings, giving brilliant and dark spots irregular in shape.

A model of diffusing and non-absorbent surface is proposed, in which the height of the scatters with respect to a reference plane are supposed as a random variable, and with a gaussian probability density function. A surface of these characteristics is, for example, a metal which is not well polished. We suppose that the rough surface is illuminated by a collimated light beam perpendicularly to its plane resulting in a speckle pattern which is calculated by means of the FFT (Fig. 7).

Due to that optical path $\delta$ followed by the different points of the wavefront is not the same, consequently, neither is the phase $2 \pi \delta / \lambda$. As we have to count the return path, the path length and height $h(x, y)$ of the surface referred to the plane $z=0$ are related by the expression $4 \pi h(x, y) / \lambda$. Thus, the reflectance will be proportional to the exponential of this phase factor, adopting the form

$$
R(x, y)=R_{0}(x, y) \exp \left(\frac{4 \pi i}{\lambda} h(x, y)\right),
$$

where $R_{0}(x, y)$ is the reflection coefficient of the surface, and $(4 \pi i h(x, y) / \lambda)$ is the phase. In the simulation presented in this paper we choose $R_{0}(x, y)=1$.

By measuring the random height $h(x, y)$ of the sampled points (Fig.8), it would be possible to construct the reflectance matrix of $N \times N$ elements. Following the nomenclature of the 


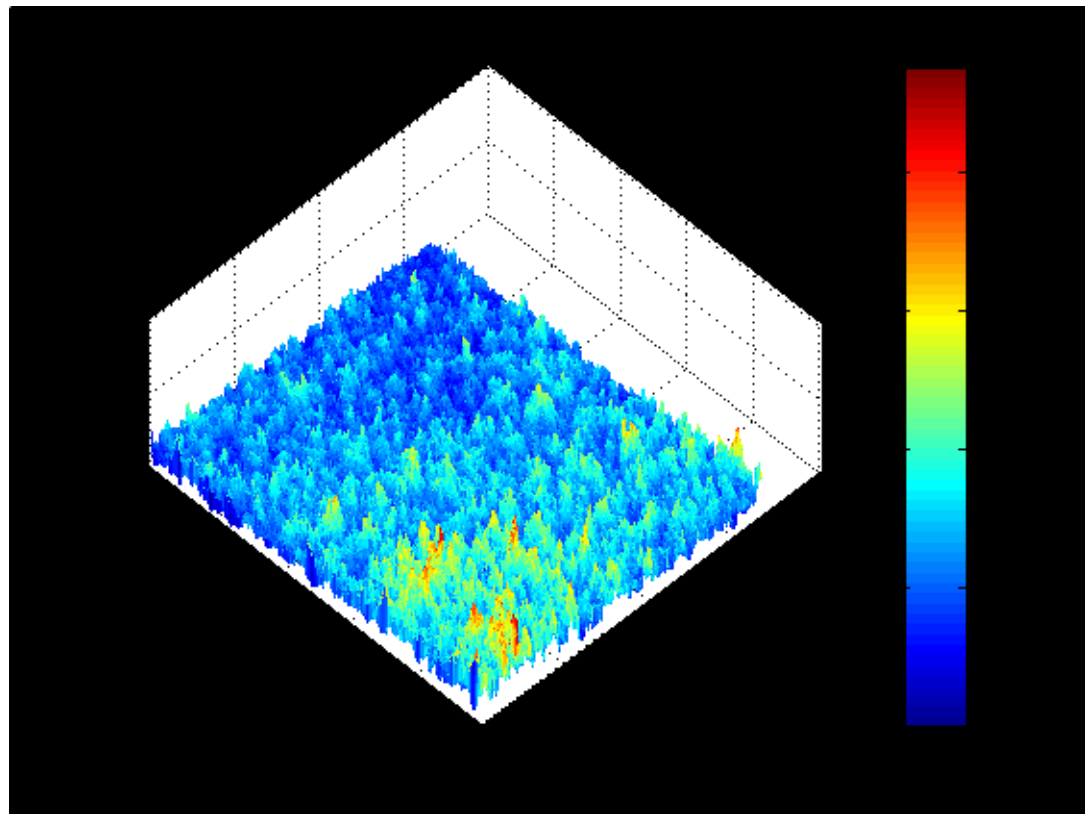

Fig. 7. Three dimensional representation of the intensity of a speckle pattern captured by a $\mathrm{CCD}$ camera in the laboratory. The values of the intensity over the $\mathrm{OZ}$ axis are in the interval $[0,255]$.

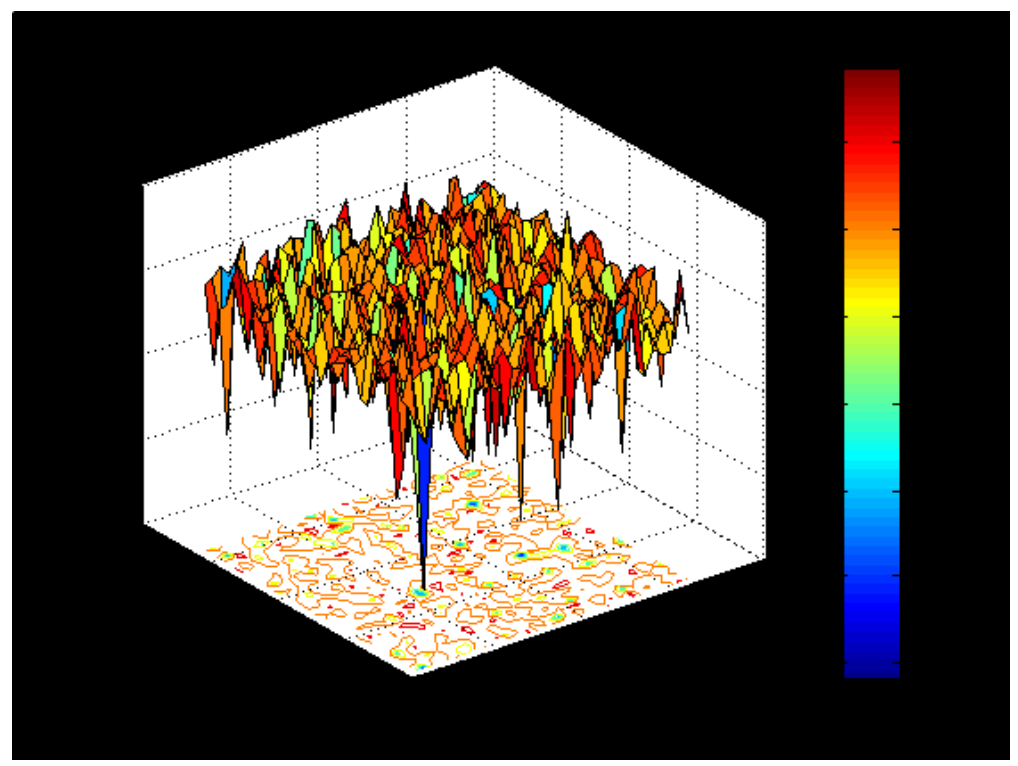

Fig. 8. Rough surface generated by MATLAB. The plane of the figure $(O X Y)$ depicts a plot of the surface contour. 
preceding section we call this array the IF matrix (Fig.9). The elements of IF contain the complex reflectance $R(x, y)$ corresponding to each point of the surface, which are separated from their neighbors a distance equal to the sampling period $u$. The area of the delimiting mask will be represented by points outside a circle with zero reflectance.

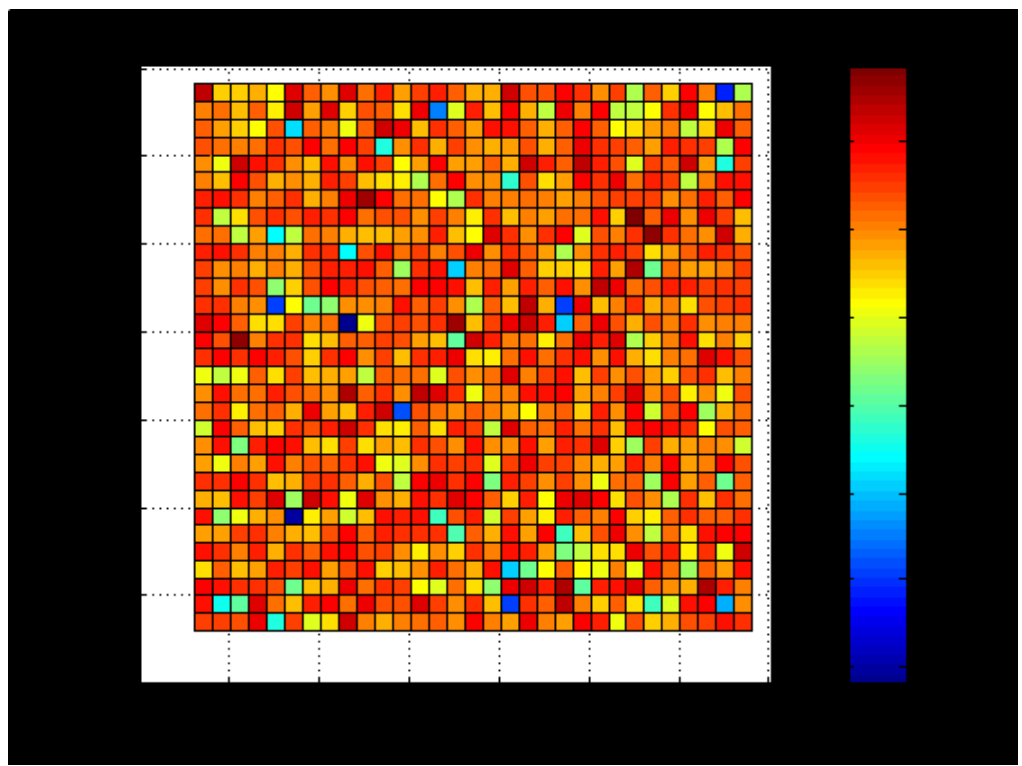

Fig. 9. View of the grid chosen on the OXY reference plane for $N=64$. The colours represent the surface heights at each point (pixel).

Following the same way as in preceding paragraphs, we employ the ratio $h / \lambda$ as a nondimensional variable, which will be very useful when changing the wavelength. In the model this variable is equal to a constant multiplied by a random number, which will provide information on the roughness in the simulation. We will call in the program this constant $R U$ and it represents a roughness modulating factor. Random numbers with Gaussian distribution are generated in MATLAB by the command randn. The mathematical expression for reflectance is

$$
R(x, y)=R_{0}(x, y) \exp \left(\frac{4 \pi i}{\lambda} R U(\operatorname{rndn}(N))\right)
$$

where

$$
\begin{gathered}
\frac{h}{\lambda}=R U \times \operatorname{randn}(N), \\
\text { and the phase } \\
\frac{\delta}{\lambda}=4 \pi \times R U \times \operatorname{randn}(N) .
\end{gathered}
$$


Thus, an element of RUrandn is a number equal to an optical path measured in wavelengths. For example, an $R U=1$ and a $r a n d n=2$ give rise $\max (R U$ randn $)=2$, which indicates a maximum path difference of $2 \lambda$, that is to say, a groove on the reflecting surface with depth equal to $\lambda$. However with the same randn, but with the modulating factor equal to 0.1 , the roughness would be a tenth part. Hence the $R U$ factor represents the roughness measured in wavelengths.

To account the transversal geometry of the incident laser beam on the surface, the rough surface is delimited by means of a round mask of diameter $D$ (geometry could be different; see section 7.2). The diameter $D$ must be greater than the wavelength and the sampling period $\mathrm{u}$. On the other hand it is supposed that the number of sampled points inside the diameter $D$ is large enough, in order be sure that the statistics applies.

Once that the characteristics of the surface and beam are defined, the diffraction pattern is obtained by means of the FFT of the reflectance matrix IF. The registered intensity of the diffracted light by the rough surface is proportional to the square modulus of the diffracted amplitude, e.g. $|F O|^{2}$.

\section{Definitions of roughness}

In this section we try to adapt some definitions of roughness to our specific problem. We start the quantitative definition of the average roughness $R_{\mathrm{a}}$ from the mean surface level, as the average absolute value of the height, for all the points along a straight line (remember the profilometer). Then in a circular matrix of diameter $D$ inside the $I F$, corresponds $\pi B D^{2} / 4$ elements. Therefore, the roughness of the sample may be expressed by the following formulae

$$
R_{a}=\frac{4 \sum_{B D}|h(x, y)|}{\pi B D},
$$

where the sum is extended to the sampled points within the circle of diameter $B D$. As previously, if we transform this Eq.(8) to non-dimensional variables, we get

$$
\frac{R_{a}}{\lambda}=\frac{4 \sum_{B D}\left|\frac{h(x, y)}{\lambda}\right|}{\pi B D}=\frac{4 \cdot R U}{\pi B D} \sum_{B D}|\operatorname{randn}(N)| .
$$

The number of elements $G$ within the beam of diameter $D(B D)$ is less than the $N^{\times} N$ elements of IF. Say $L$ the length of the square side where the surface is defined. In any case $B D=\chi L, \chi$ being a constant $(\chi \leq 1)$, then it holds

$$
G=\frac{1}{4} \pi \chi^{2}(N \times N)
$$

whose maximum value is 0.8 , approximately $(\chi=1)$. If $N$ and $B D$ are large enough, and surface heights are randomly distributed, the $G$ values are representative and $R_{\mathrm{a}}$ can be calculated using G elements. 
Similarly, the roughness $R_{\mathrm{q}}$ (root mean square) could be expressed as function of $B D$. In fact, considering the usual definition of this parameter, the following formulae may be written

$$
R_{q}=2 \cdot \sqrt{\frac{\sum_{B D}|h(x, y)|^{2}}{\pi B D}},
$$

and its non-dimensional value

$$
\frac{R_{q}}{\lambda}=\sqrt{\frac{4 \sum \frac{}{B D}\left|\frac{h(x, y)}{\lambda}\right|^{2}}{\pi B D}}=2 \cdot R U \sqrt{\frac{\sum_{B D}|\operatorname{randn}(N)|^{2}}{\pi B D}} .
$$

\section{Programming using MATLAB}

We will see that the simulated specklegram corresponding to the diffraction of a monochromatic radiation by a rough surface is altered by the roughness of the object within a certain range, which depends on the wavelength of the beam used. Therefore, by analyzing some characteristics of the intensity pattern it would be possible to measure roughness.

To understand the idea let us suppose a flat surface, well polished, delimited by an aperture (mask). If a beam strikes on the surface, the delimiting aperture diffracts it resulting in an intensity pattern that depends on the geometry of the obstacle. Now if the surface is scratched, the intensity registered changes, although the aperture maintains its geometry. In both cases the autoconvolution of the intensity is different, which means that the roughness produced on the surface is the cause of the change. Therefore, the convolution of the diffraction pattern could be indicative of the degree of surface polish.

To test the hypothesis, first we constructed a computer model of a rough surface, and second we simulate the diffraction of a collimated monochromatic beam by this surface. The resulting random intensity, that is, the speckle, is stored in a matrix (FO) and its autoconvolution $(\mathrm{CO})$ is performed. Once all data of $\mathrm{CO}$ are obtained, the functional relationship of the maximum value of the autoconvolution and its relation with the roughness is analyzed.

The program consists of the following steps:

1. Begin by setting the number of samples $N$ along each axis.

2. The matrix IF is constructed by using the command $\mathrm{RAN}=\operatorname{randn}(N)$.

3. The diameter of the laser beam $B D$ is specified, measured in number of array elements.

4. A value to the $R U$ is assigned.

5. The $B S$ array is constructed. The mask is 0 outside the circle and 1 inside.

6. The matrix RURAN $=\mathrm{RU}$ * RAN is introduced, representing the surface heights for each pixel on the area $N x N$.

7. The matrix $h s$ is defined as $h s=$ RURAN. ${ }^{*}$ BS. It represents the height of the points inside the circle (mask $M$ ).

8. The reflectance matrix is obtained. Its expression is ts $=\exp (4 \Pi i$ RURAN).

9. The array FO is calculated, which is the FFT of $t$.

10. The intensity of the diffraction pattern is determined, $F I D I=|F O|^{2}$. 
11. The autoconvolution CO of FIDI, and its maximum COV is computed.

12. In order to manipulate the data more easily, the logarithm of $C O V$ is given $(\log (C O V))$.

The detailed program may be found in appendix A

\section{Computer results}

\subsection{Circular beam}

Figures 10 shows the results of numerical calculations performed with a PC. In order to the numerical calculations are easy to obtain the data were $N=64, B D=6, R U=0,0.1,0.2,0.3$ 0.40 .5 . The successive rows of the figure refer to these values of roughness, respectively. The first column of the figure corresponds to the surface height along the diameter of the illuminated area. The second column represents the intensity of the diffraction pattern, FIDI, and the third one shows the autoconvolution, $\mathrm{CO}$.

For beginning a surface without roughness was chosen. The first row shows the area under study for a perfect mirror, illuminated by a circular beam of diameter $B D=6$. The calculated diffraction pattern shows the classical Airy disc corresponding to diffraction by a hole. With the proposed values $N=64$ and $B D=6$ is $N / B D=64 / 6$, and as $D=B D u$, gives $N u=64 D / 6$. The analysis of Figure 4 shows that the first minimum of the Airy disc in the frequency space is 24, approximately. From Fig 1 it follows

$$
\alpha_{v}=\frac{\cos \theta}{\lambda}=\frac{(v-1)}{N u} \approx \frac{(12-1)}{64 u}=\frac{11}{64 u}=0.17 \frac{1}{u} .
$$

On the other hand, the first minimum given in the theory of diffraction by a circular hole is

$$
\alpha_{v}=\frac{\cos \theta}{\lambda} \approx 1.22 \frac{1}{D u}=1.22 \frac{1}{6 u}=0.20 \frac{1}{u} .
$$

Both results agree and differ in a small amount. The difference can be attributed to the small number of values chosen.

The second row refers to the same mirror, but not completely polished, and with a coefficient $R U=0.1$. The profile shows small heights and valleys. The Airy disk is a little blurred, and not as clear as in the previous case. In the third row $R U=0.2$ the central disk appears deformed and a speckleled. In the fourth and fifth rows the figure is quit different with respect to the first one, and the speckles are on all the pattern. In the last row only speckle may be seen, and no traces of the Airy disk are present. When the roughness is $R U=$ 0.5 (bottom row) yields a rough surface with high grooves. The intensity is formed by irregular random spots being unknown directly the form of the mask, e.g. the symmetrical intensity circle of the Airy function. In summary, diffraction by a specular surface delimited by an aperture produces an intensity pattern concentrated around the direction of the reflected beam, but if the roughness is increasing, the light is diffracted producing speckle which structure is random.

The third figure of each row (third column) corresponds to autoconvolución (CO), which has a maximum at the center $(C O V)$. In effect, the values for the logarithm of $C O V$ are, respectively: $\log C O V(R U=0.0)=7.78, \log C O V(R U=0.1)=7.10, \log C O V(R U=0.2)=6.86$, $\log \operatorname{COV}(\mathrm{RU}=0.3)=6.90, \log \operatorname{COV}(R U=0.4)=6.88$, and $\log \operatorname{COV}(R U=0.5)=6.91$. In this calculus the logarithm of the autoconvolution hass been used because the maximum value of $C O$ is very large. Employing $\log (\mathrm{COV})$, the data are easier to manipulate. 

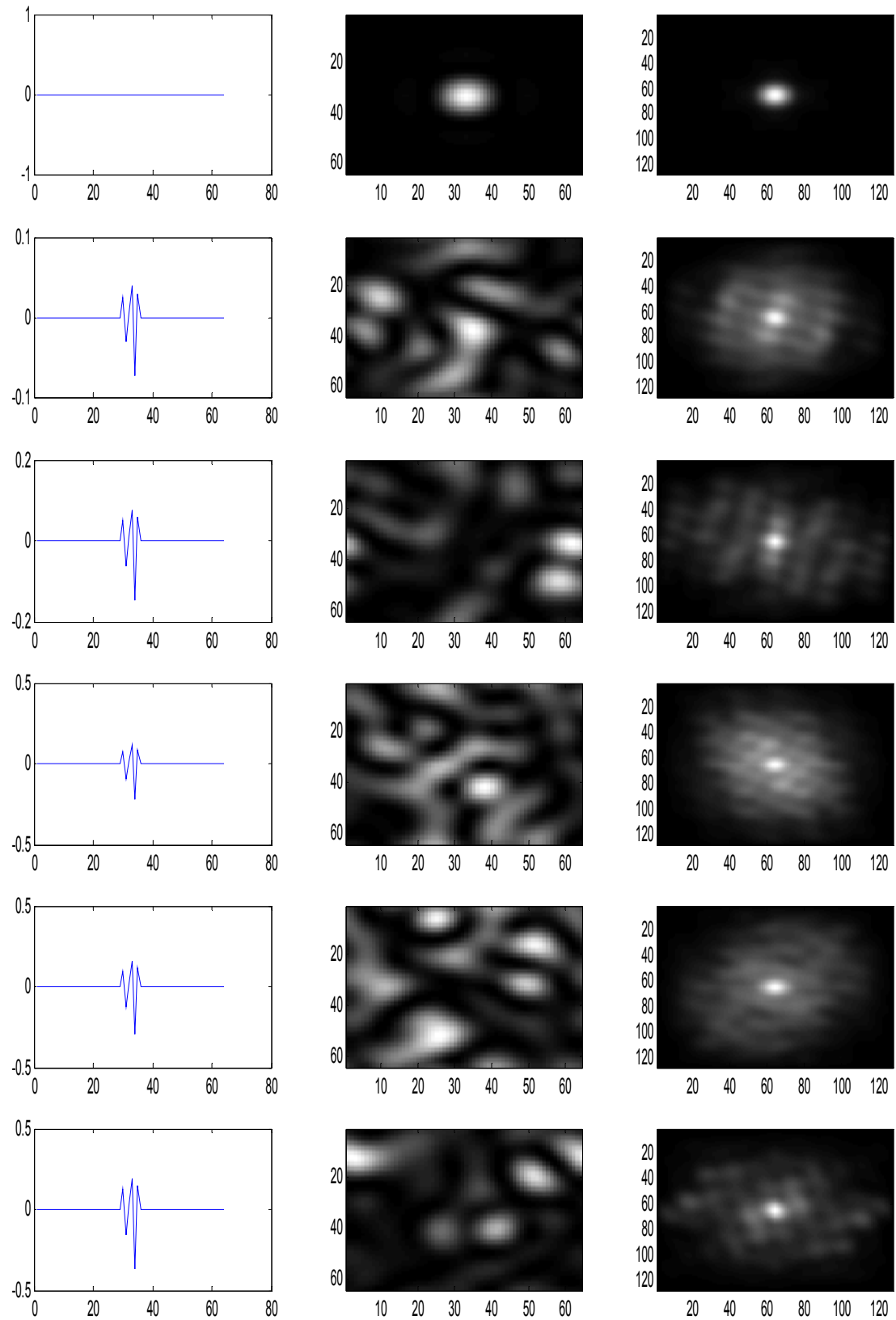

Fig. 10. Computer results for $N=64, B D=6, R U=0,0.1,0.2,0.30 .40 .5$. The first column shows the roughness along the illuminated area. The second represents the intensity of the diffraction pattern, FIDI, and the third column is the autoconvolution, $\mathrm{CO}$. The rows correspond to the different values of $R U$. 
These results show that the values of their maxima are not the same. On the contrary, the maximum value for each one depends on the surface roughness. For this reason it seems suitable to employ the maximum value of the autoconvolution of the speckle pattern, as a possible procedure for measuring the roughness of a surface, if the roughness is smaller than the wavelength of light used in the experiment.

At the same time, $C O V$ depends on the diameter of the beam used $(B D)$ also. To see the effect in the autoconvolution when the wide of the laser is changed, we computed $\log C O V$ with $N$ and $D$ for two different number of data and diameters. For example if $N=64$, and $B D$ $=32$, it yields

$$
\log C O V=12.0,11.7,10.7,9.8,9.7,9.7 \text {, }
$$

whereas with $N=128$ and $B D=32$

$$
\log C O V=12.6,12.3,11.3,10.4,10.3,10.3 .
$$

Therefore the maximum reached by the autoconvolution depends on the number of samples $N$ and the beam diameter $B D$.

This result is reasonable if we bear in mind the definition of autoconvolution. In fact, convolution may be regarded as the overlapping area between two functions (in this case the same function) when one is reversed and moves on the other. The result depends on the wide and height of the functions involved. Therefore, if the diameter of the beam changes the autoconvolution modifies its value too.

With the aim to apply this result to laboratory experiments, it seems necessary to have more values of the autoconvolution in other circumstances. In he same way as explained, the following table provide useful data of the $\log C O V$, for $N=512$ when the diameter $D$ ranges from $2^{3}$ to $2^{3+m}(m=1,2, \ldots 5)$. The detailed results for this calculation can be seen in the appendix $\mathrm{B}$.

\begin{tabular}{|c|c|c|c|c|c|c|}
\hline $\mathrm{D} \backslash \mathrm{RU}$ & 0.0 & 0.1 & 0.2 & 0.3 & 0.4 & 0.5 \\
\hline 8 & 10.233 & 9.231 & 9.148 & 9.185 & 9.078 & 9.128 \\
\hline 18 & 12.036 & 10.867 & 10.35 & 10.351 & 10.348 & 10.368 \\
\hline 32 & 13.810 & 12.558 & 11.536 & 11.546 & 11.548 & 11.542 \\
\hline 64 & 15.607 & 14.294 & 12.734 & 12.736 & 12.740 & 12.742 \\
\hline 128 & 17.412 & 16.037 & 13.945 & 13.938 & 13.941 & 13.937 \\
\hline 256 & 19.216 & 17.838 & 15.149 & 15.141 & 15.145 & 15.142 \\
\hline 512 & 21.257 & 19.881 & 16.428 & 16.347 & 16.347 & 16.349 \\
\hline
\end{tabular}

Table 1. Values of the autoconvolution $\log (\mathrm{COV})$ for different diameters and roughness parameters. The results in yellow do not give information since they are very similar.

From these results may be inferred that if the area of the illuminated surface is known, measuring experimentally the autoconvolution of the speckle pattern, it would be possible measuring the roughness of this surface. To conduct laboratory experiments would be necessary to build larger tables with more values, for different incident beam intensities.

By examining the calculations it also follows that for values of $R U$ close to zero, the difference between $\log C O V$, corresponding to a $B D$, and a diameter corresponding to half value, is approximately constant and equal to 2 , i.e. 


$$
\log C O V^{\prime}-\log C O V=2 \quad=>C^{\prime} O V^{\prime} / C O V=10^{2},
$$

and, in general,

$$
\frac{\operatorname{COV}\left(N^{\prime}\right)}{\operatorname{COV}(N)}=10^{\left(\frac{N^{\prime}}{N}\right)} .
$$

This property will be important for ulterior calculations.

\subsection{Square mask}

In the preceding developing calculations, a circular geometry for the beam was supposed. However, other possibilities may occur. For instance, when a laser ray is directed onto a sample under an angle of incidence $\theta$, the effective area intersected by the beam has a quasielliptical form. Although an elliptical mask is easy to simulate with MATLAB, this paragraph deals with the study of the effect of employing a square aperture. This approximation simplifies the program, since there is no need the beam diameter datum. Moreover, from the point of view of the results, it has little influence in the final values when comparing these values with those obtained for an elliptical mask.

The simulation gives the results of $\log (C O V)$ for $R U=0.0,0.1, \ldots .0 .5$, and $N=16,32,64,128$, 256,512 , that appear in the following table:

\begin{tabular}{|c|c|c|c|c|c|c|}
\hline $\mathrm{N} \backslash$ RU & 0.0 & 0.1 & 0.2 & 0.3 & 0.4 & 0.5 \\
\hline 16 & 9.63 & 9.24 & 8.08 & 7.52 & 7.59 & 7.57 \\
\hline 32 & 12.04 & 11.72 & 10.73 & 9.49 & 9.33 & 9.34 \\
\hline 64 & 14.45 & 14.10 & 13.05 & 11.47 & 11.14 & 11.14 \\
\hline 128 & 16.86 & 16.51 & 15.48 & 13.81 & 12.95 & 12.95 \\
\hline 256 & 19.27 & 18.92 & 17.89 & 16.19 & 14.80 & 14.75 \\
\hline 512 & 21.67 & 21.33 & 20.30 & 18.57 & 16.68 & 16.56 \\
\hline
\end{tabular}

Table 2. Values of the autoconvolution $\log (\mathrm{COV})$ for different data and roughness parameter $R U$. The results in green do not give information.

Figure 11 represents the values of the attached table II. These curves show the dependence of $\log (\mathrm{COV})$ with the roughness for different values of $N$, provide that the roughness is less than 0.4. Therefore, the trend is maintained even if the aperture is different. From the figure it follows that, except for values marked in green on the table, the dependence of $\log C O V$ with roughness is approximately parabolic, and can be approximated by the equation

$$
\log C O V=a+b \times R U^{2} .
$$

As in section 7.1., the difference of the $\log (C O V)$ for consecutive values of $R U$, follows certain regularity. In fact, if the values of $\log (C O V)$ for $R U=0$ are examined (see figure 11), we observe that for adjacent values of this variable, the differences between two consecutive points (corresponding to double $N$ ) are: 2.41, 2.41,2.41, 2.41, 2.40. Taking into consideration these differences, the following mathematical relationship is verified: 


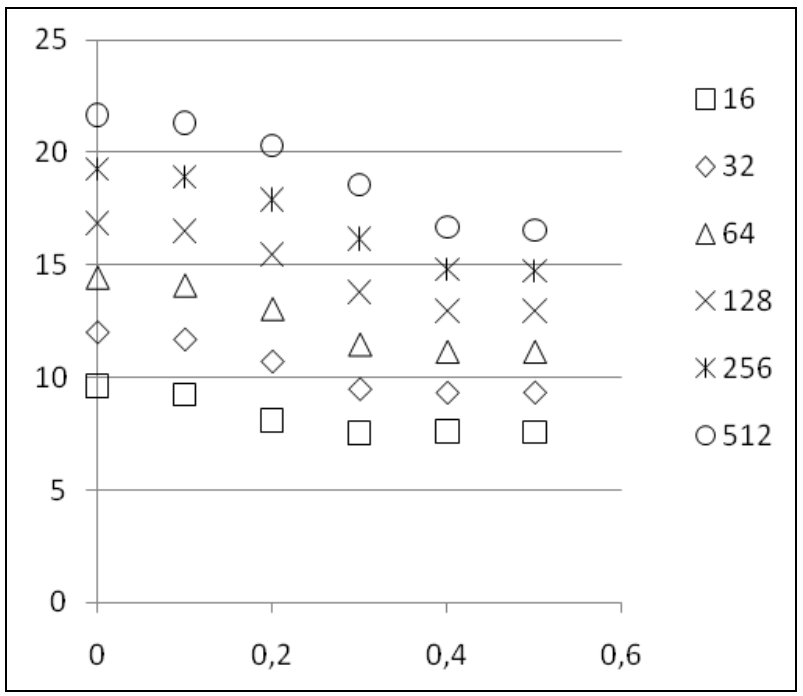

Fig. 11. Values of $\log (C O V)$ for roughness $R U=0.0,0.1, \ldots 0.5$.

$$
\log C O V^{\prime}-\log C O V=2.41 \Rightarrow \frac{C O V^{\prime}}{C O V}=10^{2.41}=257 \approx 2^{8} \quad \forall N^{\prime} / N \approx 2 .
$$

Table 3 below relates the difference of logarithms with the ratio $N^{\prime} / N$.

\begin{tabular}{|c|c|c|c|c|c|c|}
\hline $\mathrm{N}^{\prime} / \mathrm{N}$ & 1 & 2 & 4 & 8 & $\ldots \ldots$ & $2^{\mathrm{n}}$ \\
\hline $\log \mathrm{COV}^{\prime}-\log \mathrm{COV}$ & 0 & 2.41 & $2 \times 2.41$ & $3 \times 2.41$ & $\ldots \ldots$ & $\mathrm{n} \times 2.41$ \\
\hline
\end{tabular}

Table 3. Logarithmic difference for $R U=0$

From these values it yields,

$$
\frac{N^{\prime}}{N}=2^{n} \Rightarrow n=\frac{\log \left(N^{\prime} / N\right)}{\log 2}
$$

therefore,

$$
\begin{aligned}
& \log C O V^{\prime}-\log C O V=2.41 \frac{\log \left(N^{\prime} / N\right)}{\log 2} \Rightarrow \log \frac{C O V^{\prime}}{C O V}= \\
& \log \left(N^{\prime} / N\right)^{2.41 / \log 2}=\log \left(N^{\prime} / N\right)^{7.999} \approx \log \left(N^{\prime} / N\right)^{8}
\end{aligned}
$$

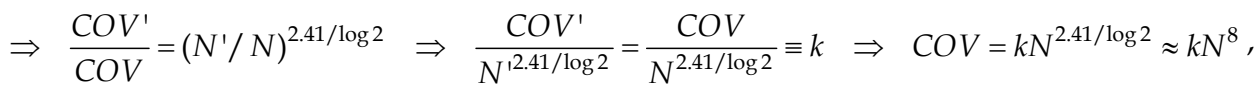

where $k$ is a constant. From the definition of $k$ it follows that

$$
\log k=\log C O V-\frac{2.41}{\log 2} \log N .
$$

Applying this formula for $N=64$ we have 


$$
\log k=14.45-\frac{2.41}{\log 2} \log 64=-0.01000 \Rightarrow k=0.9772,
$$

therefore,

$$
\log C O V=-0.01+\frac{2.41}{\log 2} \log N=-0.01+8.006 \log N .
$$

Using this result to the values of $N: 32,64,256,512(R U=0)$, we btain for $\log (C O V)$ : 12.04, $14.45,16.86,19.27$, and 21.68 , respectively. These results agree with those of the table II.

A more general fit for $\log (C O V)$ considering and $R U$ can be found, provided that $R U \leq 0.4$ :

$$
\log C O V=-0.01000+8.006 \log N+b \times R U^{2} .
$$

To determine the value of $b$, we choose, for example, $N=128$ and $R U=0.2$, which yields

$$
\begin{aligned}
15.48 & =-0.01000+8.006 \log 128+b \times 0.2^{2} \Rightarrow b=-34.50 \\
\Rightarrow \log C O V & =-0.01000+8.006 \log N-34.50 R U^{2} \approx 8.006 \log N-34.50 R U^{2} .
\end{aligned}
$$

Solving the unknown in Eq. (20) we have

$$
R U=\sqrt{\frac{1}{34.5} \log \left(\frac{N^{8}}{C O V}\right)} .
$$

The advantage of this formula is that it allows calculating the value of the roughness for each $N$ and $D$.

To verify the accuracy of these results, we introduce some values of roughness and number of samples in Eq.(20).

For $R U=0.1, N=32$ :

$$
\log C O V=8.006 \log 32-34.50 \times 0.1^{2}=11.70
$$

For $R U=0.1, N=512$ :

$$
\log C O V=8.006 \log 512-34.50 \times 0.1^{2}=21.35
$$

For $R U=0.3, N=32$ :

$$
\log C O V=8.006 \log 32-34.50 \times 0.3^{2}=8.95
$$

For $R U=0.3, N=512$ :

$$
\log C O V=8.006 \log 512-34.50 \times 0.3^{2}=18.59 .
$$

It may be seen that the differences between these values calculated with the formula (20) and those displayed in table II are equal to or less than 0.02 , except for the case $N=32, R U=$ 0.3 , which is 0.15 . But as in this case the value of the table does not correspond to the difference of logarithms (marked in green), it follows that the equation obtained is suitable for the specified intervals. 


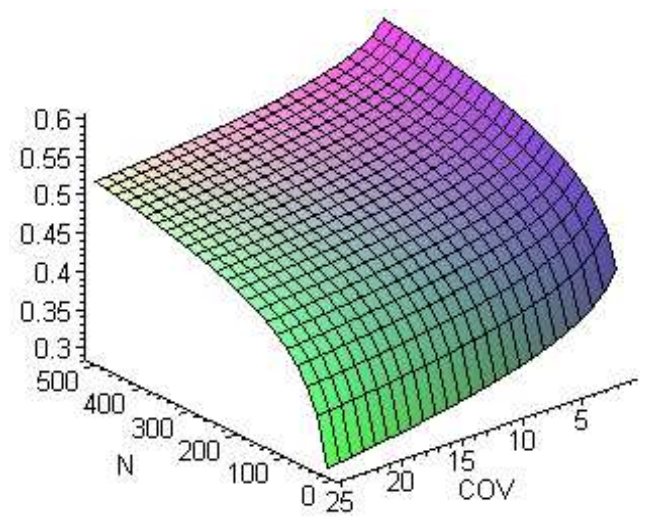

Fig. 12. Values of $R U$ (from 0.0 to 0.5 ) as a function of $N$ and $C O V$ (Eq.(22)).

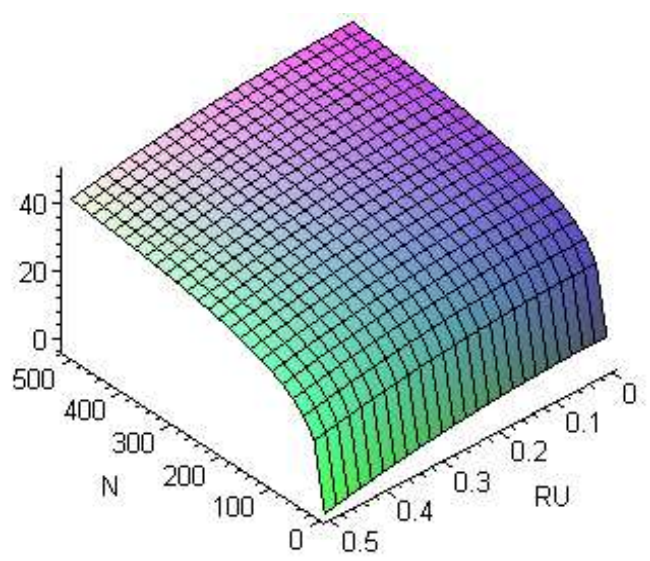

Fig. 13. Thissurface represents the function $\log (\mathrm{COV})$ for differerent values of $\mathrm{N}$ and $R U$ (Eq.(21)).

\section{Apendix A}

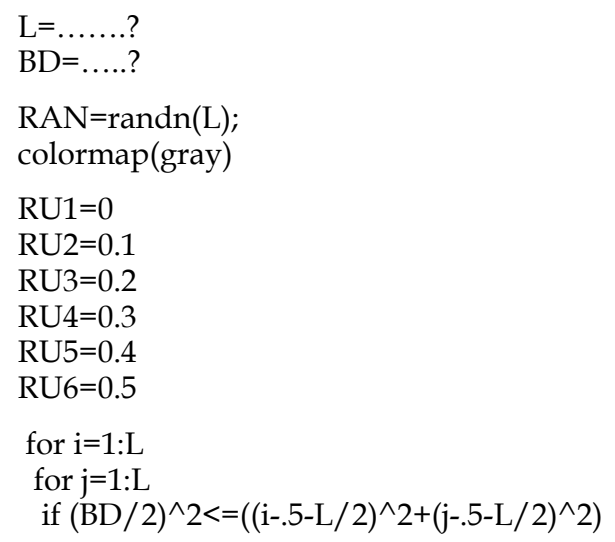




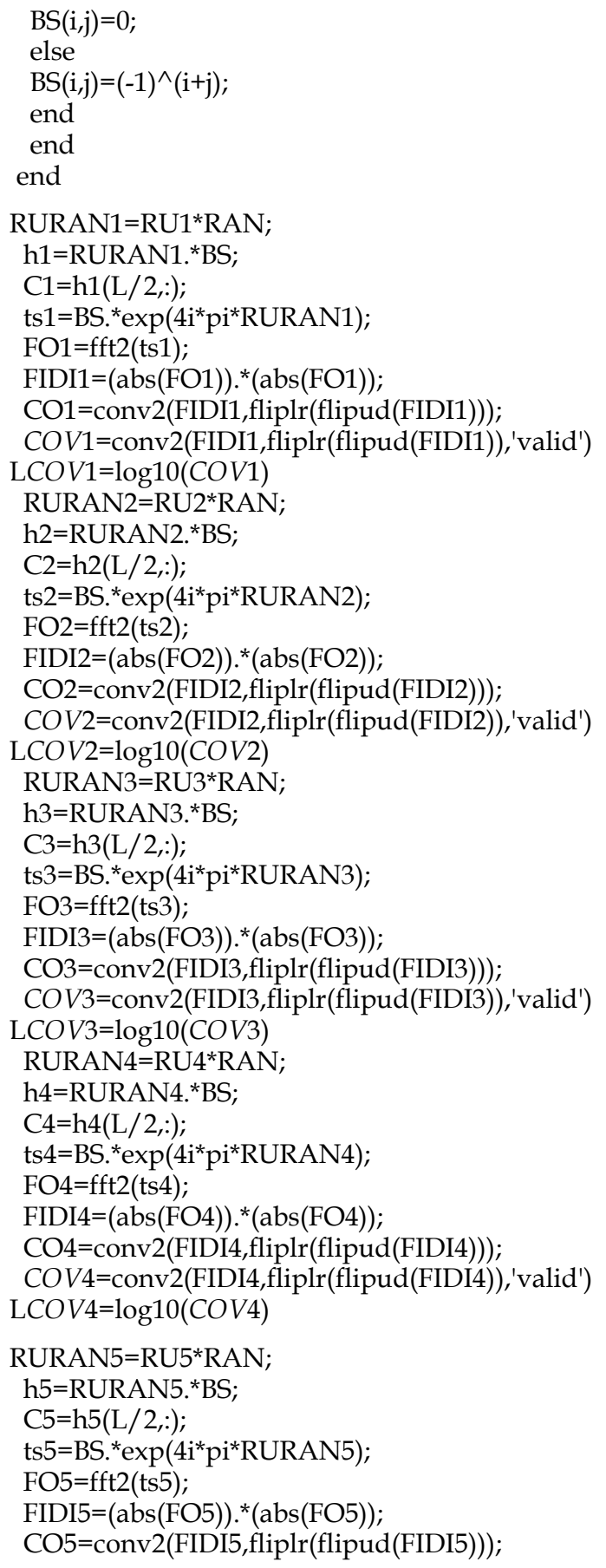




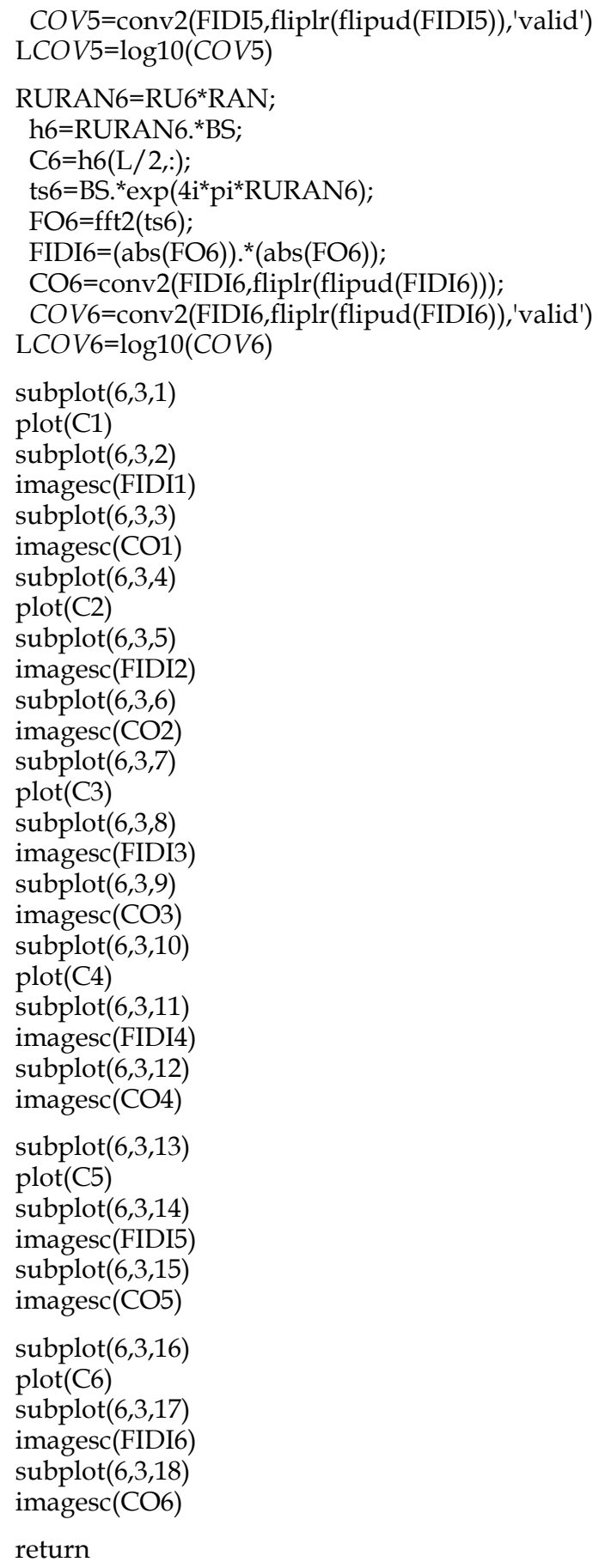




\section{Apendix B}
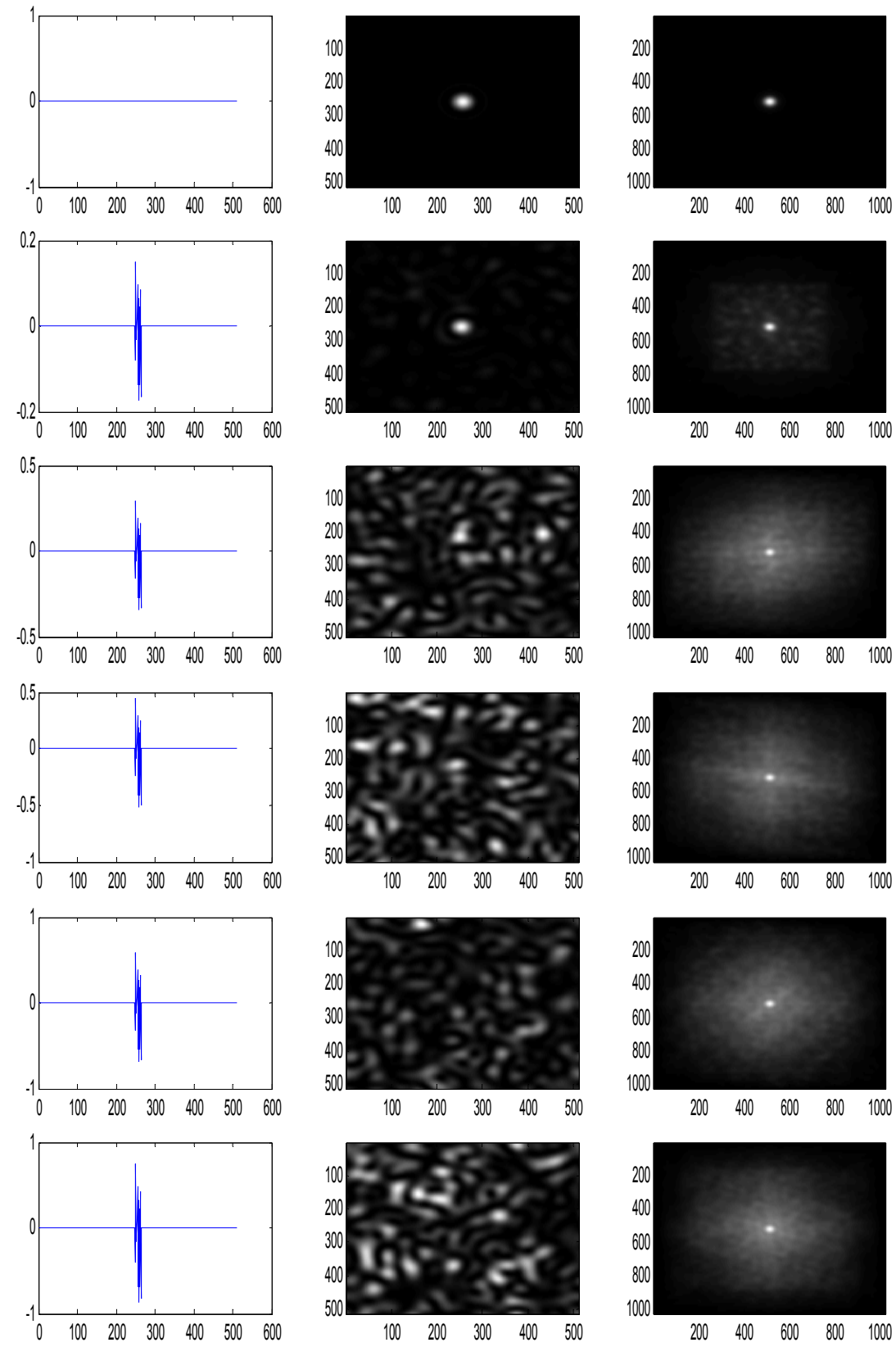

Fig. 14. $N=500 ; D=8 ; R U=0.0,0.1,0.2,0.3,0.4,0.5$. (a) Surface height along the diameter. (b) Diffraction pattern. (c) Autoconvolution. 


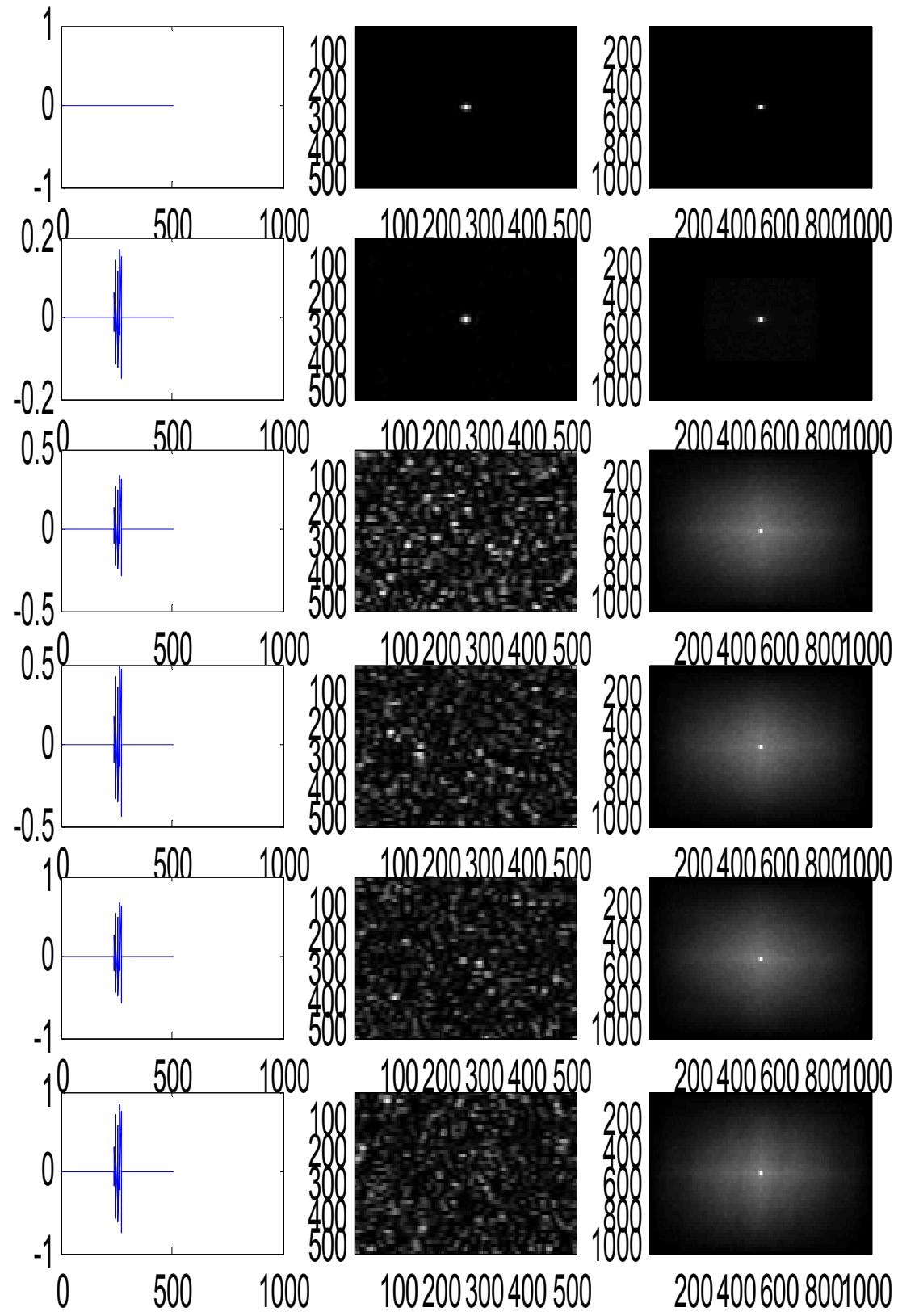

Fig. 15. $N=500 ; D=16 ; R U=0.0,0.1,0.2,0.3,0.4,0.5$. (a) Surface height along the diameter. (b) Speckle pattern. (c) Autoconvolution. 


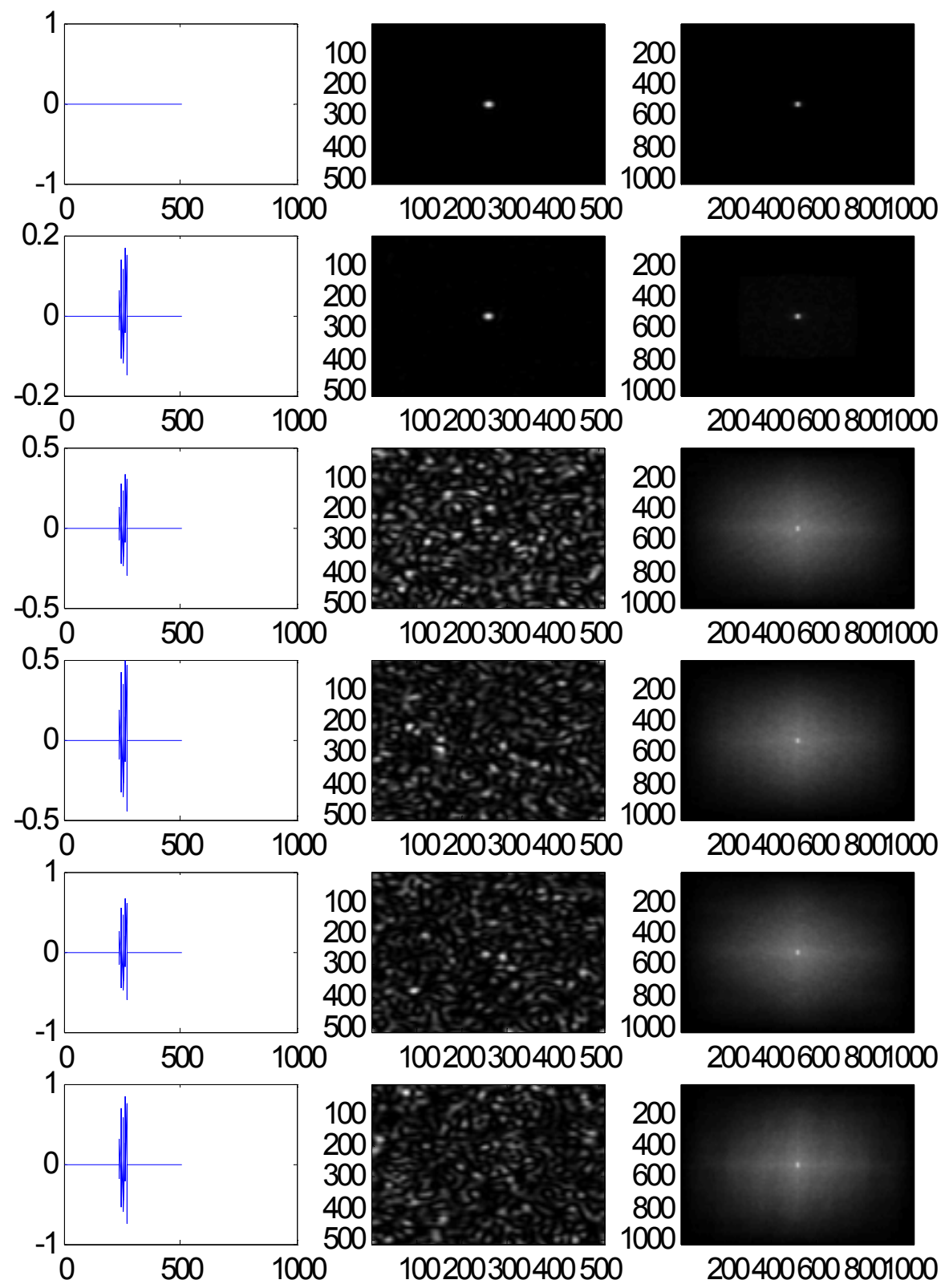

Fig. 16. $N=500 ; D=32 ; R U=0.0,0.1,0.2,0.3,0.4,0.5$. (a) Surface height along the diameter. (b) Speckle pattern. (c) Autoconvolution. 

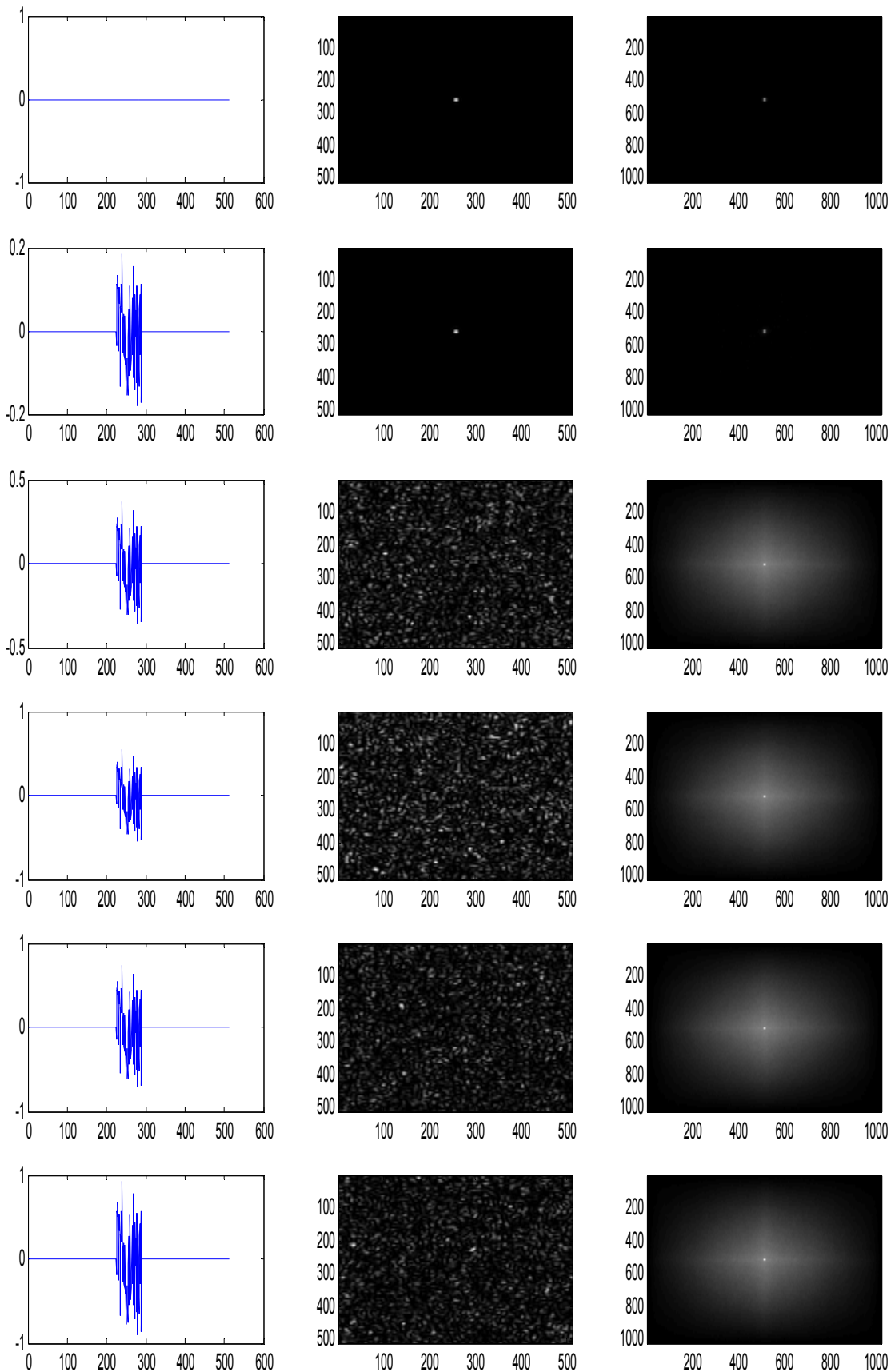

Fig. 17. $N=500 ; D=64 ; R U=0.0,0.1,0.2,0.3,0.4,0.5$. (a) Surface height along the diameter. (b) Speckle pattern. (c) Autoconvolution. 

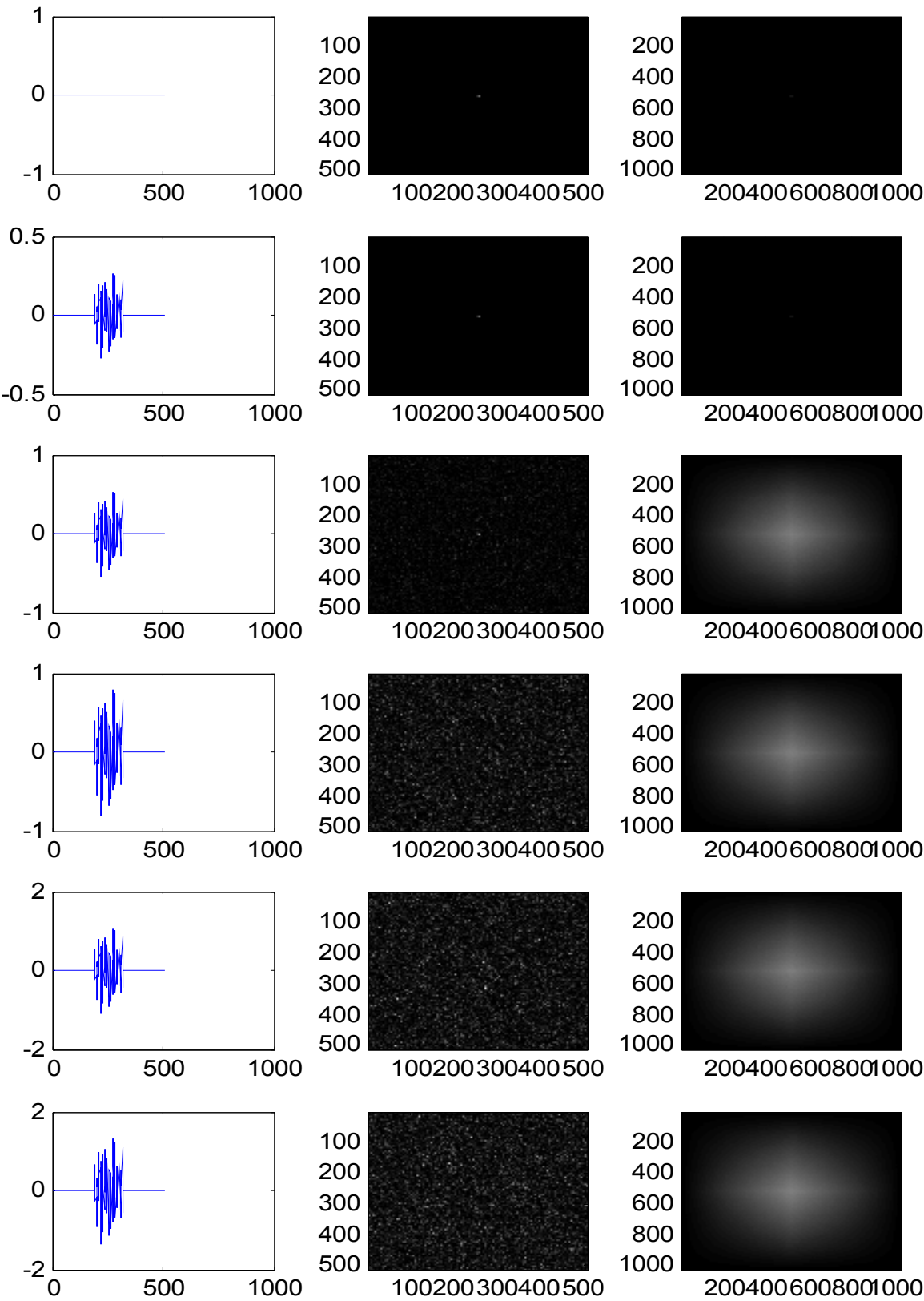

2004006008001000

Fig. 18. $N=500 ; D=128 ; R U=0.0,0.1,0.2,0.3,0.4,0.5$. (a) Surface height along the diameter. (b) Speckle pattern. (c) Autoconvolution. 

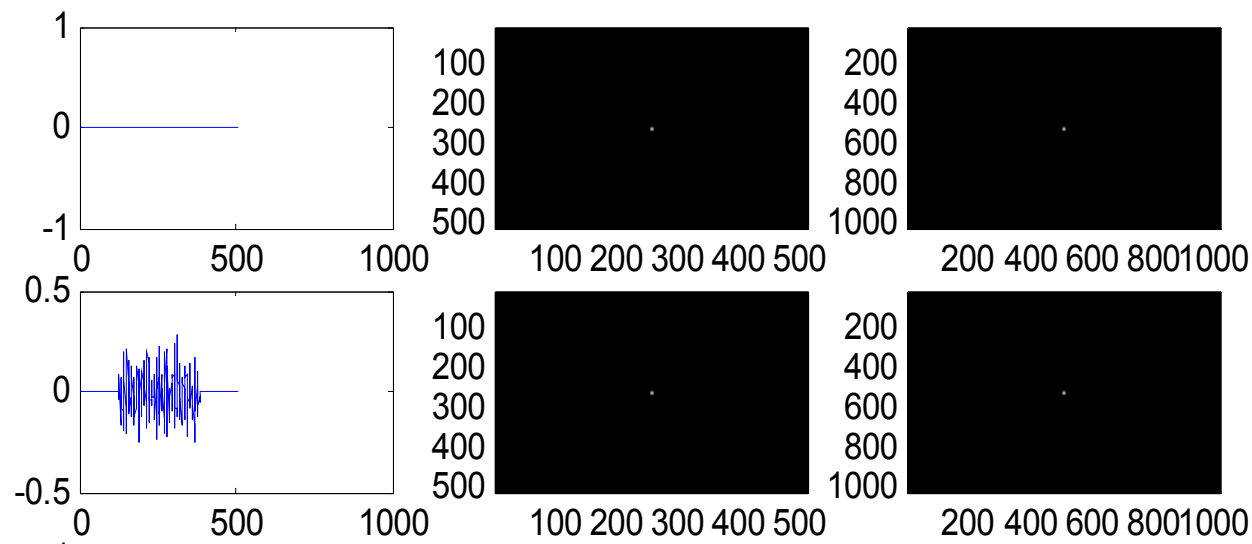

100200300400500

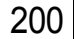

400

600

800 1000
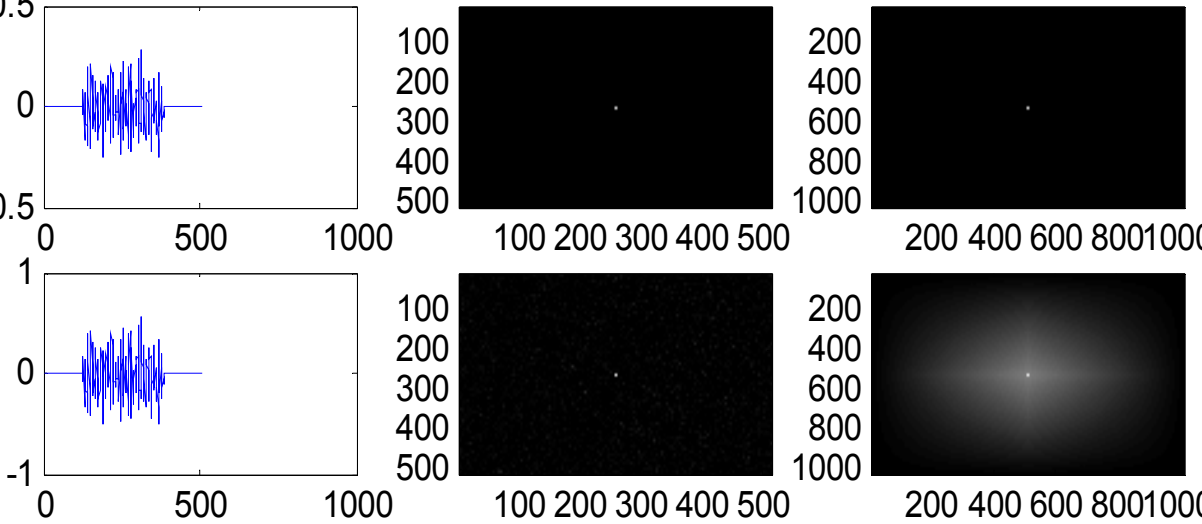

100

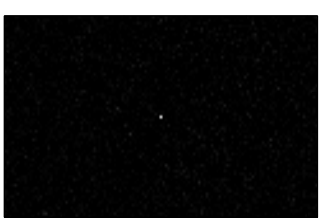

200

400

600

800

400

1000

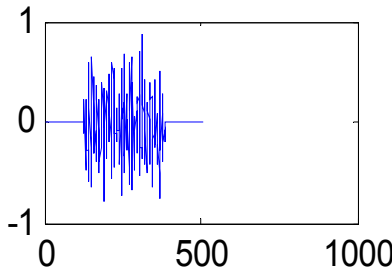

100200300400500

2004006008001000
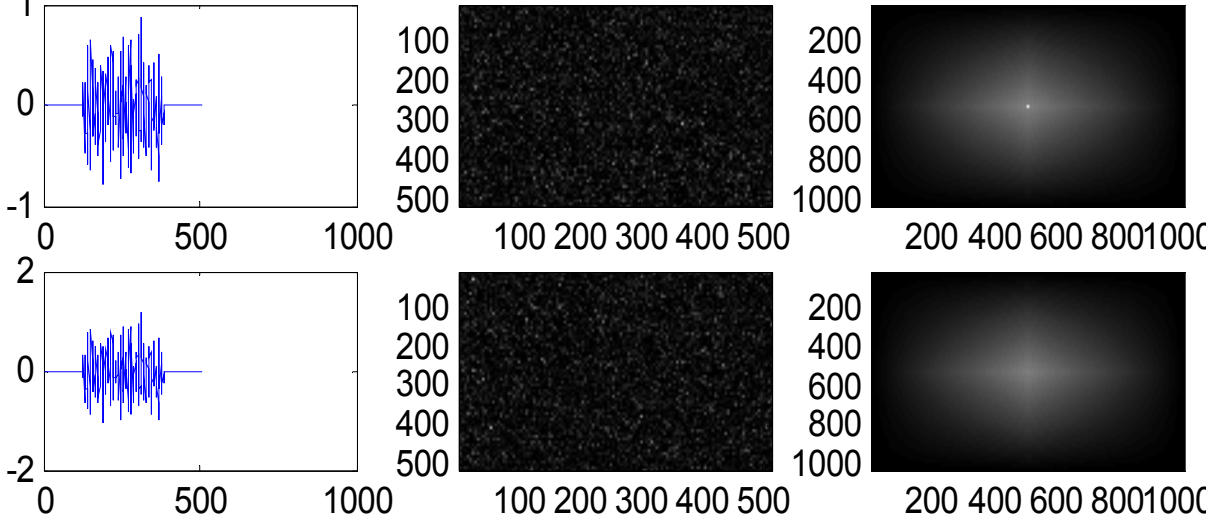

100
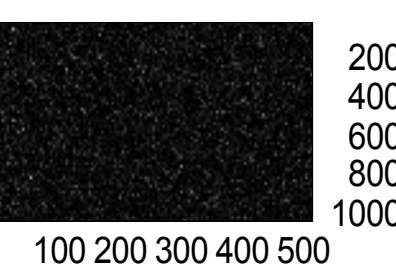

2004006008001000
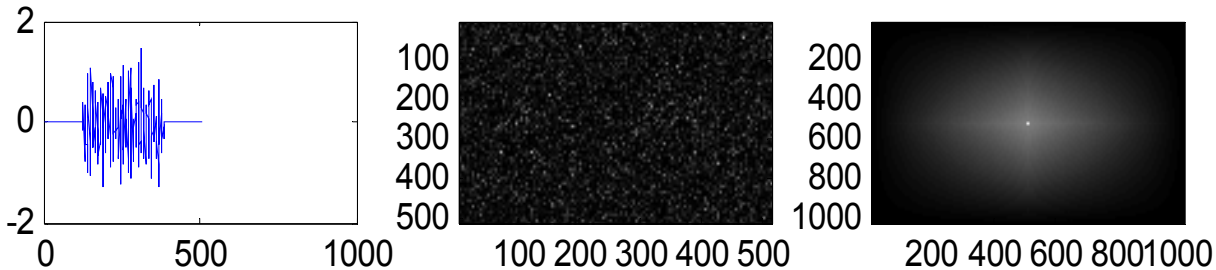

Fig. 19. $N=500 ; D=256 ; R U=0.0,0.1,0.2,0.3,0.4,0.5$. (a) Surface height along the diameter. (b) Speckle pattern. (c) Autoconvolution. 

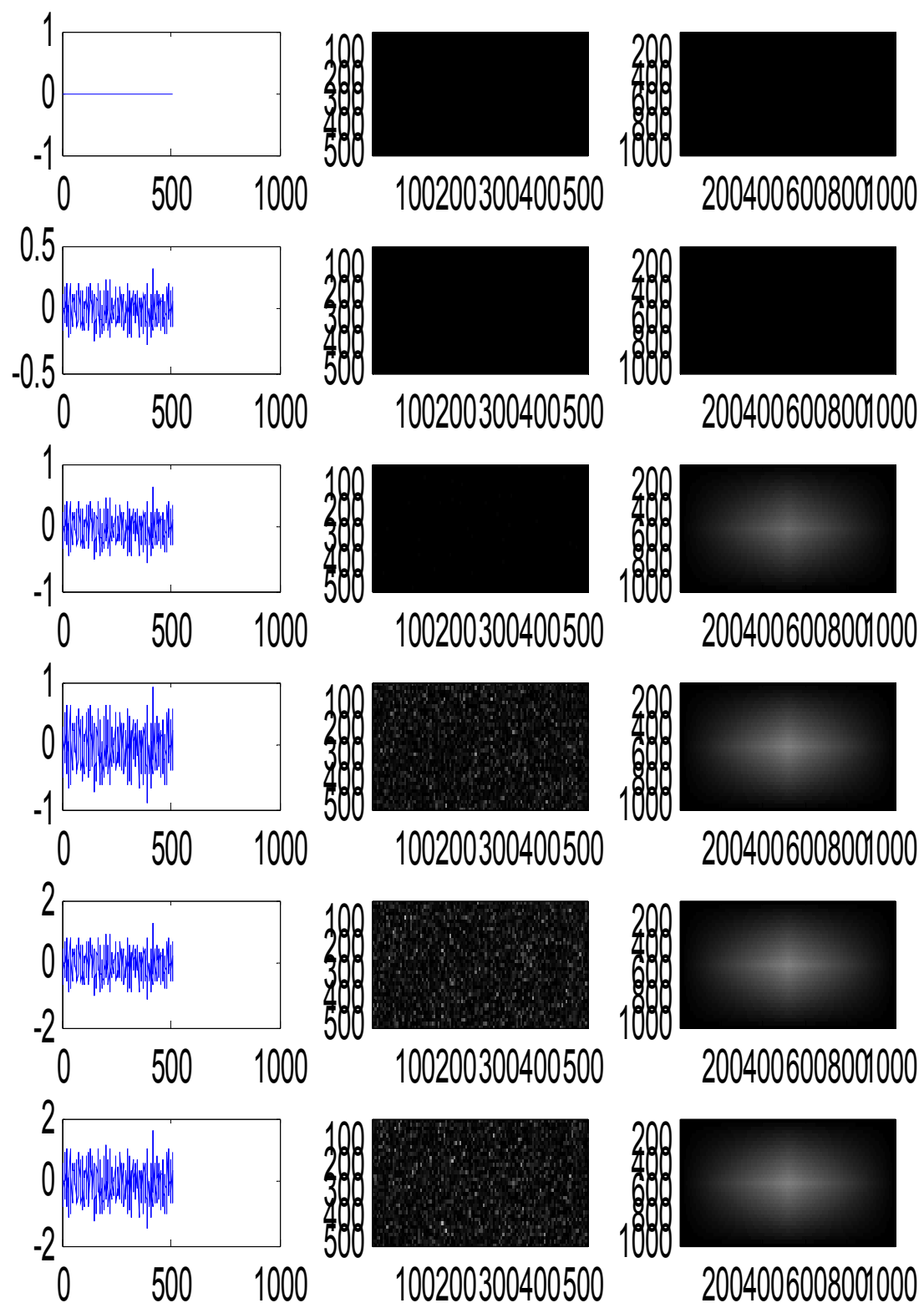

Fig. 20. $N=500 ; D=500 ; R U=0.0,0.1,0.2,0.3,0.4,0.5$. (a) Surface height along the diameter. (b) Speckle pattern. (c) Autoconvolution. 


\section{References}

Born, M; Wolf, E; (1999) Principles Optics, Cambridge University Press. pp. 412-484.

Etter, D.M.; (1997) Engineering problem solving with MATLAB. Prentice-Hall.

Gascón, F.; Salazar, F.; (2006) A simple method to simulate diffraction and speckle patterns with a PC, Optik, Vol. 117, pp. 49-57.

Gascón, F.; Salazar, F.; (2008) Numerical computation of in-plane displacements and their detection in the near field by double-exposure objective speckle photography, Opt. Commun., Vol. 281, pp- 6097-6106.

Glio, M.; Musazzi, S.; Perini, U.; Surface measurement by means of speckle wavelength decorrelation", Opt. Commun. Vol. 28, 1979, pp. 166-170.

Goodman, J.W.; (1975) Dependence of image speckle contrast on surface roughness. Opt. Commun, Vol. 14, pp. 324-327.

Huntley, J.M.; (1989) Speckle photography fringe analysis: assessment of current algorithms, Appl. Opt. Vol. 28, pp. 4316-4322. (See references therein).

Kreis, T.; (2005) Handbook of Holographic Interferometry. Wiley-VCH, Weinheim, Ch.1, 2.

Lehmann, P.; Patzelt, S.; Schöne, A.; (1997) Surface roughness measurement by means of polychromatic speckle elongation. Appl. Opt. Vol. 36, pp. 2188-2197.

Leonhardt, K.; Tiziani , H.J.; (1982) Removing ambiguities in surface roughness measurement. Optica Acta, Vol. 29, pp. 493-499

Lipson, S.G.; Lipson, H.; (1995) Tannhauser: Optical Physics. Cambridge University Press, Cambridge, p.162.

Patzelt, S.; Horn, F; Goch, (2006) G; Fast integral optical roughness measurement of specular reflecting surfaces in the nanometer range. XVIII Imeko World Congress, Rio de Janeiro, Brazil.

Persson, U.; (2006) Surface roughness measurement on machined surfaces using angular speckle correlation. J. Mater. Process. Tech., Vol. 180, pp. 233-238.

Pearson, U.; (1993) Measurement of surface roughness on rough machined surfaces using spectral speckle correlation and image analysis. Wear, Vol. 160, pp. 221-225.

Pérez Quintián, F., Rebollo, M.A; Nogert, E.N.; Landau M. R.; Gaggioli, N.G.; (1996) Relationship between speckle correlation and refraction index variations: applications for roughness measurements", Opt. Eng. Vol 35, , pp. 1175-1178.

Ruffing, B.; (1986) Application of speckle-correlation methods to surface-roughness measurement: a theoretical study, J. Opt. Soc. Am. A, vol. 3, pp. 1297-1304.

Ruffing, B.; (1987) Non-contacting roughness measurement of technical surfaces by specklecorrelation method. Doctoral Thesis. University of Karlsruhe. (In german)

Spagnolo, G.S.; Paoletti, D.; (1996) Digital speckle correlation for on-line real-time measurement. Opt. Commun. Vol. 132, pp. 24-28.

Stratton, J.A.: (1961) Théorie de l'électromagnétisme, Dunod, Paris. p. 531

Tay, C. J.; Toh, S. L.; Shang, H. M.; Zhang, J.; (1995) Whole-field determination of surface roughness by speckle correlation. Appl. Opt, vol. 34, pp. 2324-2335.

Xiaomei, Xu.; (2009) Non-contact Surface Roughness Measurement Based on Laser Technology and Neural Network. Proc. IEEE, International Conference on Mechatronics and Automation. Changchun, China.

Yamaguchi, I.; Kobayashi, K.; Yaroslavsky, L.; (2004) Measurement of surface roughness by speckle correlation. Opt. Eng., Vol. 43, pp. 2753-2761. 
Yoshimura, T.; Kato, K.; Nakagawa, K.; (1990) Surface-roughness dependence of the intensity correlation function under speckle-pattern illumination, J. Opt. Soc. Am. A, Vol. 7, pp. 2254-2259.

Zhao, Gao; Xuezeng, Zhao; (2008) On-Line Surface Roughness Measurement Based on Specular Intensity Component of Speckle Patterns. Proc. IEEE 2008, International Conference on Information and Automation. Zhangjiajie, China. 


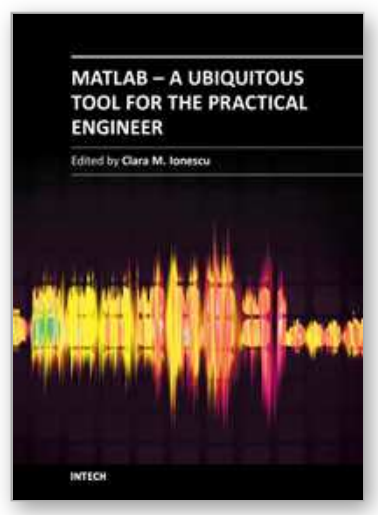

\section{MATLAB - A Ubiquitous Tool for the Practical Engineer}

Edited by Prof. Clara Ionescu

ISBN 978-953-307-907-3

Hard cover, 564 pages

Publisher InTech

Published online 13, October, 2011

Published in print edition October, 2011

A well-known statement says that the PID controller is the â€œbread and butterâ€ of the control engineer. This is indeed true, from a scientific standpoint. However, nowadays, in the era of computer science, when the paper and pencil have been replaced by the keyboard and the display of computers, one may equally say that MATLAB is the â€œbreadâ€ in the above statement. MATLAB has became a de facto tool for the modern system engineer. This book is written for both engineering students, as well as for practicing engineers. The wide range of applications in which MATLAB is the working framework, shows that it is a powerful, comprehensive and easy-to-use environment for performing technical computations. The book includes various excellent applications in which MATLAB is employed: from pure algebraic computations to data acquisition in real-life experiments, from control strategies to image processing algorithms, from graphical user interface design for educational purposes to Simulink embedded systems.

\section{How to reference}

In order to correctly reference this scholarly work, feel free to copy and paste the following:

F. Gascón and F. Salazar (2011). Simulation of Rough Surfaces and Analysis of Roughness by MATLAB, MATLAB - A Ubiquitous Tool for the Practical Engineer, Prof. Clara Ionescu (Ed.), ISBN: 978-953-307-907-3, InTech, Available from: http://www.intechopen.com/books/matlab-a-ubiquitous-tool-for-the-practicalengineer/simulation-of-rough-surfaces-and-analysis-of-roughness-by-matlab

\section{INTECH}

open science | open minds

\section{InTech Europe}

University Campus STeP Ri

Slavka Krautzeka 83/A

51000 Rijeka, Croatia

Phone: +385 (51) 770447

Fax: +385 (51) 686166

www.intechopen.com

\section{InTech China}

Unit 405, Office Block, Hotel Equatorial Shanghai

No.65, Yan An Road (West), Shanghai, 200040, China

中国上海市延安西路65号上海国际贵都大饭店办公楼 405 单元

Phone: +86-21-62489820

Fax: +86-21-62489821 
(C) 2011 The Author(s). Licensee IntechOpen. This is an open access article distributed under the terms of the Creative Commons Attribution 3.0 License, which permits unrestricted use, distribution, and reproduction in any medium, provided the original work is properly cited. 